\title{
A Mechanistic Analysis of Trimethylanilinium Salt Degradation: Implications for Methylation and Cross-coupling Applications
}

\author{
Jack B. Washington, ${ }^{a}+$ Michele Assante, ${ }^{b}+$ Chunhui Yan, ${ }^{a}$ David McKinney, ${ }^{a}$ Vanessa Juba, ${ }^{a}$ Andrew \\ G. Leach, ${ }^{\mathrm{c}}$ Sharon E. Baillie, ${ }^{\mathrm{d}}$ and Marc Reid*a \\ $\mathrm{N}, \mathrm{N}, \mathrm{N}$-trimethylanilinium salts are known to display dual reactivity through both the aryl group and the $\mathrm{N}$-methyl groups. \\ These salts have thus been widely applied in cross-coupling, aryl etherification, fluorine radiolabelling, phase-transfer \\ catalysis, supramolecular recognition, polymer design, and (more recently) methylation. However, their application as \\ electrophilic methylating reagents remains somewhat underexplored, and an understanding of their arylation versus \\ methylation reactivities is lacking. This study presents a mechanistic degradation analysis of $N, N, N$-trimethylanilinium salts \\ and highlights the implications for synthetic applications of this important class of salts. Kinetic degradation studies, in both \\ solid state and solution phases, have delivered insights into the physical and chemical parameters affecting anilinium salt \\ stability. ${ }^{1} \mathrm{H}$ NMR kinetic analysis of salt degradation has evidenced thermal degradation to methyl iodide and the parent \\ aniline, consistent with a closed-shell $\mathrm{S}_{\mathrm{N}} 2$-centred degradative pathway, and methyl iodide being the key reactive species in \\ applied methylation procedures. Furthermore, the effect of halide and non-nucleophilic counterions on salt degradation has \\ been investigated, along with deuterium isotope and solvent effects. Finally, new mechanistic insights have enabled the \\ investigation of the use of trimethylanilinium salts in O-methylation and in improved cross-coupling strategies.
}

\section{Introduction}

Applications and divergent reactivity of trimethylanilinium salts.

$\mathrm{N}, \mathrm{N}, \mathrm{N}$-trimethylanilinium salts have found wide-ranging applications in synthesis, spanning phase-transfer catalysis, ${ }^{1}$ supramolecular ion-pairing catalysis, ${ }^{2,3}$ host-guest binding studies, ${ }^{4}$ O-methylation, ${ }^{5}$ O-arylation, ${ }^{6}$ heteroatom arylations, ${ }^{7}$ $\mathrm{C}-\mathrm{H}$ methylation, ${ }^{8}$ C-arylation, ${ }^{9-11}$ fluorine radiolabeling, ${ }^{12-15}$ organometallic ligand design, ${ }^{16-21}$ antimicrobial polymer design, ${ }^{22}$ and a range of metal-catalysed cross-coupling methodologies. ${ }^{23-29}$ The dichotomy of arylation versus methylation reactivity, while important for optimising the above-listed applications, is rarely studied in detail. Understanding the dual reactivity of $N, N, N$-trimethylanilinium salts thus serves as the focus of our study.

Arylation methodologies are arguably the most widely studied of the above-listed anilinium salt applications. Pioneering developments by Wenkert et al. involved a nickelcatalysed Kumada-type coupling between Grignard reagents and trimethylanilinium iodides to generate biaryls via $\mathrm{C}-\mathrm{C}$ bond formation (Figure 1a)..${ }^{9}$ Later developments from Reeves used trimethylanilinium triflates and aryl Grignard reagents with a palladium catalyst to produce functionalised biaryl motifs in high yields under mild conditions. ${ }^{10}$ Similarly, MacMillan et al. found that trimethylanilinium triflates were suitable coupling partners with arylboronic acids in Suzuki reactions. ${ }^{11}$ Other notable examples where trimethylanilinium salts have been used as electrophilic coupling partners include Negishi coupling, ${ }^{30}$ borylation, ${ }^{31,32}$ amination, ${ }^{33}$ and azole arylation. ${ }^{34}$ In contrast to the wealth of transition-metal catalysed arylation reactions using anilinium salts, Chatani's team has demonstrated that $N, N, N$-trimethylanilinium iodide can be used to transfer a methyl (as opposed to phenyl) group in nickelcatalysed $\mathrm{C}\left(\mathrm{sp}^{2}\right)-\mathrm{H}$ and $\mathrm{C}\left(\mathrm{sp}^{3}\right)-\mathrm{H}$ bond formations (Figure $\mathbf{1 b}$ ). ${ }^{8}$ Base-mediated reactions with anilinium salts display a similar dichotomy in arylation versus methylation (Figure 1c-d). To optimise the predictable use of trimethylanilinium salts in synthesis, a structured mechanistic analysis of their arylation and methylation reactivities is required. Herein, we present our investigation of the factors governing this dichotomous reactivity (Figure 1, bottom).

\section{Drivers towards understanding the methylating ability of} anilinium salts

The presence or absence of small methyl groups in organic molecules can bring about large changes in physicochemical properties. ${ }^{35,36}$ This has been a particular focus in drug design where the installation of methyl groups can have drastic effects on solubility, potency and selectivity; this contested phenomenon is dubbed the magic methyl effect. ${ }^{37,38}$ As such, the present study to understand trimethylanilinium salts is, in 


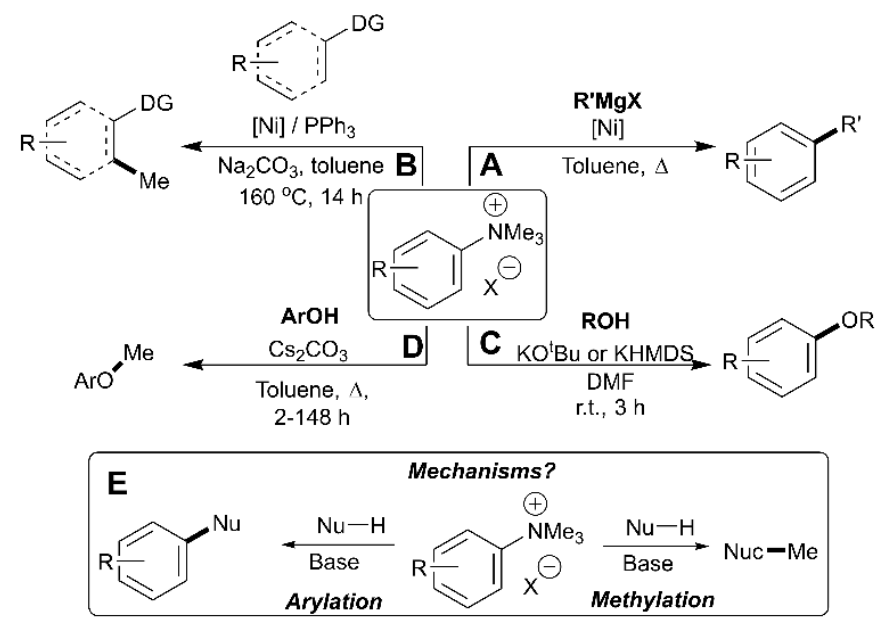

Figure 1. Use of trimethylanilinium salts in: (A) Kumada coupling, ${ }^{9}$ (B) C-H methylation, ${ }^{8}$ (C) alcohol arylation, ${ }^{6}$ and (D) phenol methylation. Part (E) summarises the dual reactivity of trimethylanilinium salts and the key mechanistic problem in this study.

part, an answer to the call for new methylation reactions. ${ }^{37}$ Parallel to drug design, formal access to highly toxic electrophilic methyl halide reagents in situ via anilinium salts may provide safer alternatives to more widely known methylating reagents. ${ }^{39}$

Of the various classes of methylating reagent available, ${ }^{40-43}$ electrophilic sources still represent a significant tool for synthetic chemists (Figure 2). As with all reagents, toxicity, safety, scalability, and operational difficulty play a key role in the decision to use or replace said reagent. ${ }^{39}$ Within the electrophilic class of methylating reagents, quaternary ammonium salts are somewhat underexplored. ${ }^{41}$ Early examples applied tetraalkylammonium and trimethylanilinium hydroxides and halides to achieve heteroatom-alkylations. ${ }^{5,44-}$ 47 From the patent literature, anilinium salts have been used to achieve O-methylation in alkaloids. ${ }^{48-50}$

To the above-mentioned design and safety-focussed ends, methods that allow for methylation of a substrate in a straightforward, safe, and predictable manner are highly desirable. Simultaneously, this directed investigation of trimethylanilinium salts, and specifically their degradation behaviour, can provide strategic insights for the myriad of aforementioned applications of these salts.

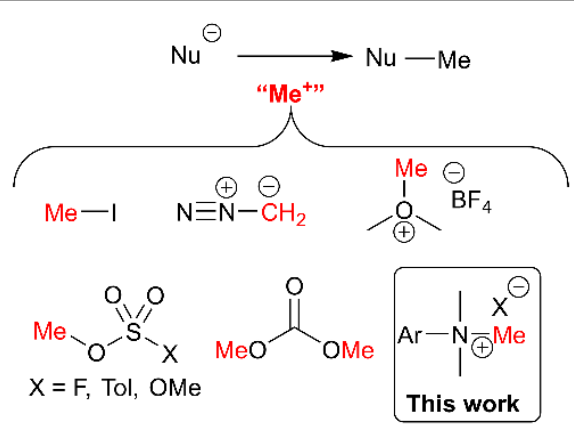

Figure 2. Commonly used electrophilic methylating reagents and the focus of this study to understand the place of trimethylanilinium salts among them .

\begin{tabular}{llll:llll} 
Salt & $\mathbf{R}$ & $\mathbf{X}$ & $\mathbf{Y}$ & $\mathbf{S a l t}$ & $\mathbf{R}$ & $\mathbf{X}$ & $\mathbf{Y}$ \\
\hline $\mathbf{1 a}$ & $\mathrm{H}$ & $\mathrm{CH}$ & $\mathrm{I}$ & $\mathbf{8 a}$ & $4-\mathrm{OMe}$ & $\mathrm{CH}$ & $\mathrm{I}$ \\
$\mathbf{1 b}$ & $\mathrm{H}$ & $\mathrm{CH}$ & $\mathrm{Br}$ & $\mathbf{9 a}$ & $3-\mathrm{OMe}$ & $\mathrm{CH}$ & $\mathrm{I}$ \\
$\mathbf{1 c}$ & $\mathrm{H}$ & $\mathrm{CH}$ & $\mathrm{Cl}$ & $\mathbf{1 0 a}$ & $3-\mathrm{Cl}$ & $\mathrm{CH}$ & $\mathrm{I}$ \\
$\mathbf{2 a}$ & $\mathrm{H}$ & $\mathrm{N}$ & $\mathrm{I}$ & $\mathbf{1 1 a}$ & $3-\mathrm{Br}$ & $\mathrm{CH}$ & $\mathrm{I}$ \\
$\mathbf{3 a}$ & $4-\mathrm{CHO}$ & $\mathrm{CH}$ & $\mathrm{I}$ & $\mathbf{1 2 a}$ & $4-\mathrm{Me}$ & $\mathrm{CH}$ & $\mathrm{I}$ \\
$\mathbf{4 a}$ & $4-\mathrm{Bz}$ & $\mathrm{CH}$ & $\mathrm{I}$ & $\mathbf{1 3 a}$ & $3-\mathrm{Me}$ & $\mathrm{CH}$ & $\mathrm{I}$ \\
$\mathbf{5 a}$ & $4-\mathrm{F}$ & $\mathrm{CH}$ & $\mathrm{I}$ & $\mathbf{1 4 a}$ & $2-\mathrm{Me}$ & $\mathrm{CH}$ & $\mathrm{I}$ \\
$\mathbf{6 a}$ & $4-\mathrm{Cl}$ & $\mathrm{CH}$ & $\mathrm{I}$ & $\mathbf{1 5 a}$ & $4-\mathrm{CF}_{3}$ & $\mathrm{CH}$ & $\mathrm{I}$ \\
$\mathbf{7 a}$ & $4-\mathrm{Br}$ & $\mathrm{CH}$ & $\mathrm{I}$ & & & &
\end{tabular}

Figure 3. Library of $N, N, N$-trimethylarylammonium (phenyl and pyridyl) salts used in mechanistic degradation studies. Further anionic variations are also considered.

\section{Results \& Discussion}

\section{Solid phase degradation analysis}

To understand the potential for long-term safe storage of trimethylanilinium salts, 51 our studies began with an investigation of the solid-state stability of arylammonium halides (primarily aniline derivatives). Salts $\mathbf{1 a - 1 c}$ and $\mathbf{2 a - 1 5 a}$ composed the core structural library employed in our investigations (Figure 3). Details on the synthesis and availability of all salts are available in the Experimental Supporting Information (Section 3).

Thermal gravimetric analysis (TGA) was used to estimate relative thermal stabilities of the salt library over $40-300{ }^{\circ} \mathrm{C}$ under both argon and air atmospheres. TGA traces for the argon experiments are shown in Figure 4. Detailed onset and peak degradation temperatures are shown in the Experimental Supporting Information Section 4. All salts tested were stable up to $165^{\circ} \mathrm{C}$, suggesting that storage of these compounds at room temperature would be viable. TGA traces in air were comparable to TGA results obtained under argon.

In general, compounds with more electron deficient aryl groups were more susceptible to thermal degradation (Figure 5). All salts, with the exception of $1 \mathbf{c}, 3 \mathbf{3 a}$ and $4 \mathbf{a}$, showed a smooth, single-step TGA trace. It seemed plausible that the

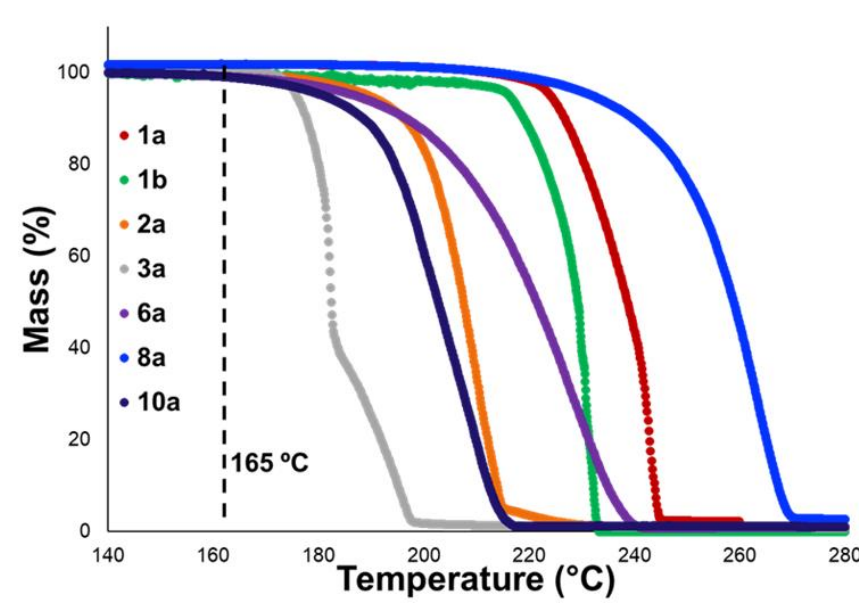

Figure 4. Mass vs. temperature trace determined via thermal gravimetric analysis (TGA) for an illustrative subset of the salt library, analysed under an argon atmosphere. Temperature range: $40-300{ }^{\circ} \mathrm{C}$. Temperature ramp: $10{ }^{\circ} \mathrm{C} / \mathrm{min}$. Temperatures between $40-139^{\circ} \mathrm{C}$ and $>280^{\circ} \mathrm{C}$ have been omitted as no observable change in mass was observed for any of the salts in these temperature ranges. 


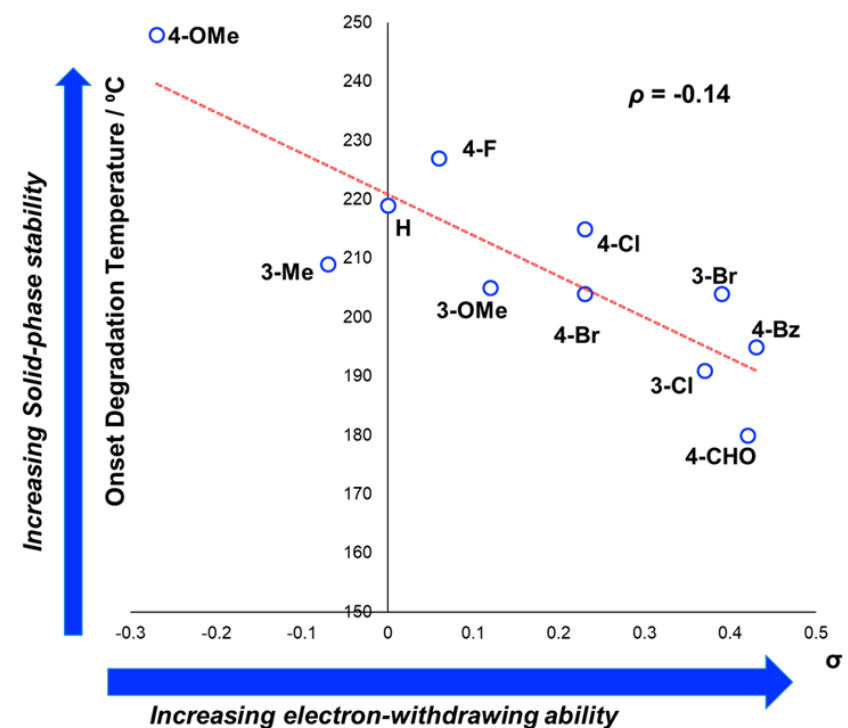

Figure 5. TGA analysis for the degradation of anilinium iodide salts, supporting the role of electron-withdrawing aryl substituents in promoting thermal degradation Reaction Additional analyses for samples degraded under air can be found in the $\mathrm{SI} .{ }^{52}$

main salt decomposition products would be the parent functionalised amino arene and its respective methyl halide, arising from a retro-Menshutkin reaction. ${ }^{52}$ This hypothesis was consistent with the observation that more electron-poor salts degraded at lower temperatures. Complementary thermal volumetric analysis (TVA $)^{53}$ coupled with a sub-ambient distillation apparatus (TVA-SAD) was used to monitor the decomposition of anilinium iodide salts $\mathbf{1 a}, \mathbf{7 a}$ and $\mathbf{1 2 a}$. Alongside IR analysis, the TVA-SAD studies provided evidence that methyl iodide was generated during the thermal decomposition of these trimethylanilinium iodide salts (see Experimental Supporting Information Section 5). It is important to note that the relative position of an electrophilic N-Me group and a halide counterion arising from crystal packing in the solid state could contribute to different relative thermal stabilities.

\section{Single-point solution phase degradation studies - substituent and} anion effects

After investigating the stability of the $\mathrm{N}, \mathrm{N}, \mathrm{N}$ trimethylanilinium iodide library in the solid state, our attention turned to investigating their degradation behaviour in solution. DMSO was strategically chosen as a solvent due to its ability to solubilise the salts, enabling homogeneous ${ }^{1} \mathrm{H}$ NMR kinetic analysis. Additionally, the high boiling point of DMSO allowed for flexible temperature studies. A graph showing the extent of degradation of each salt after a 20 min period is shown in Figure 6. Consistent with TGA analysis, electron-poor salts were the most susceptible to thermal degradation in DMSO (Figure 7); salts with a predominantly resonance electron-withdrawing 4substituent, 4-formyl (3a) and 4-benzoyl (4a), were shown to degrade by $83 \pm 6 \%$ and $70 \pm 6 \%$, respectively, and significantly more so than the unsubstituted iodide $1 \mathrm{a}$ at $16 \pm 2 \%$. Salts containing 4-chloro $(6 a ; 30 \pm 11 \%)$ and 4-bromo (7a; 39 $\pm 5 \%)$ substituents were only slightly destabilised with respect to $\mathbf{1 a}$, whilst 4-methyl (12a; $6 \pm 1 \%$ ) was more stable than $1 \mathbf{a}$. Halogen

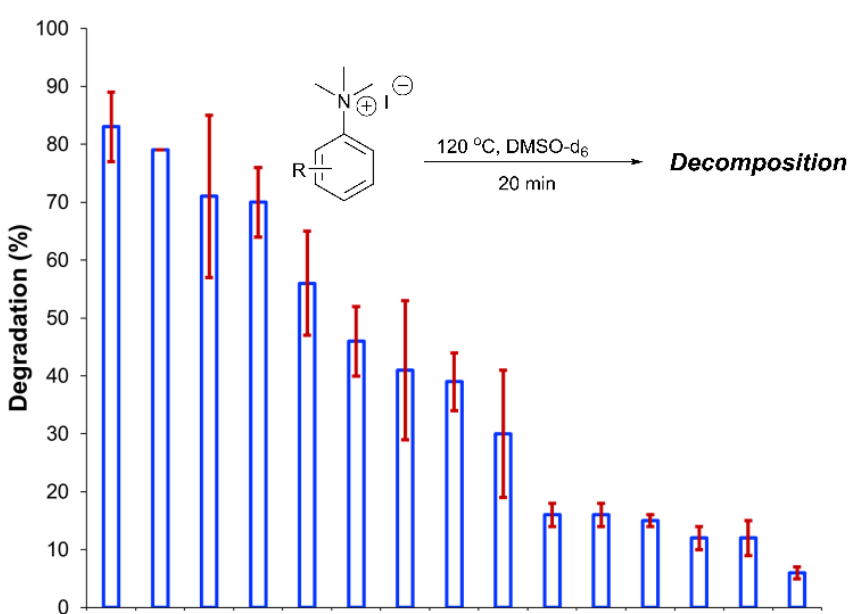

3a 15a 14a 4a 2a 10a 11a 7a 6a 9a 1a 8a 5a 13a 12a

Figure 6. Relative degradation of a range of $N, N, N$-trimethylanilinium iodides upon heating in DMSO- $\mathrm{d}_{6}(0.06 \mathrm{M})$ at $120^{\circ} \mathrm{C}$ for $20 \mathrm{~min} ; 1,2,4,5$-tetramethylbenzene was used as an internal standard to calculate the concentration of the anilinium salt before and after heating. Results and associated errors are calculated from triplicate runs.

substituents have inductive electron withdrawing effects which would destabilise the anilinium cation, but they also have the competing ability to stabilise this charge through resonance donation; ${ }^{54}$ the trend in decreasing stability from 5 a to $7 \mathbf{a}(4-\mathrm{F}>$ $4-\mathrm{Cl}>4-\mathrm{Br}$ ) is consistent with decreasing resonance donation down the group. Conversely, $4-\mathrm{CF}_{3}(\mathbf{1 5 a} ; \mathbf{7 9 \pm 0 \% )}$ shows that a strong inductive electron-withdrawing effect significantly destabilises the anilinium salt versus the unsubstituted 1a. 2Me (14a; 71 $\pm 1 \%)$ shows high anilinium salt degradation over 20 min, and can presumably be attributed to the steric encumberment of the $o$-Me and resulting destabilisation of the reactive quaternary nitrogen centre.

Beyond substitution effects of the aromatic moiety on $N, N, N$-trimethylanilinium iodide stability, we next investigated the counter-anion effect on solution-phase stability. The degradations of $N, N, N$-trimethylanilinium iodide (1a), bromide (1b), and chloride (1c) were analysed after heating to $120^{\circ} \mathrm{C}$ for 20 min in DMSO- $d_{6}$ (Figure 8). A clear trend emerged where degradation was more advanced after $20 \mathrm{~min}$ in 1c (chloride; $85 \pm 2 \%$ ) than $\mathbf{1 b}$ (bromide; $42 \pm 2 \%$ ) and 1 a (iodide; $23 \pm 3 \%$ ). This is consistent with the reported order of halide nucleophilicity in polar aprotic solvents ${ }^{55,56}$ As described below, the reaction in which methyl halide is formed is close to thermoneutrality and thus small changes in the stability of ion pairs and of carbon halide bonds can provide a thermodynamic explanation for the observed order. ${ }^{12,57}$

Understanding the effect of additives alongside salt degradation is relevant for applications of these anilinium salts in cross-coupling methodologies. ${ }^{11-17}$ To this end, an equimolar quantity of selected halide salts was added to solutions of $1 \mathrm{a}$ in DMSO- $d_{6}$ and the degradation measured over $20 \mathrm{~min}$ at $120^{\circ} \mathrm{C}$ (Figure 9). Anilinium degradation was only accelerated by 3 of the 10 additives tested, namely $\mathrm{LiCl}$, and tetrabutylammonium (TBA) salts of fluoride and chloride. The more pronounced 


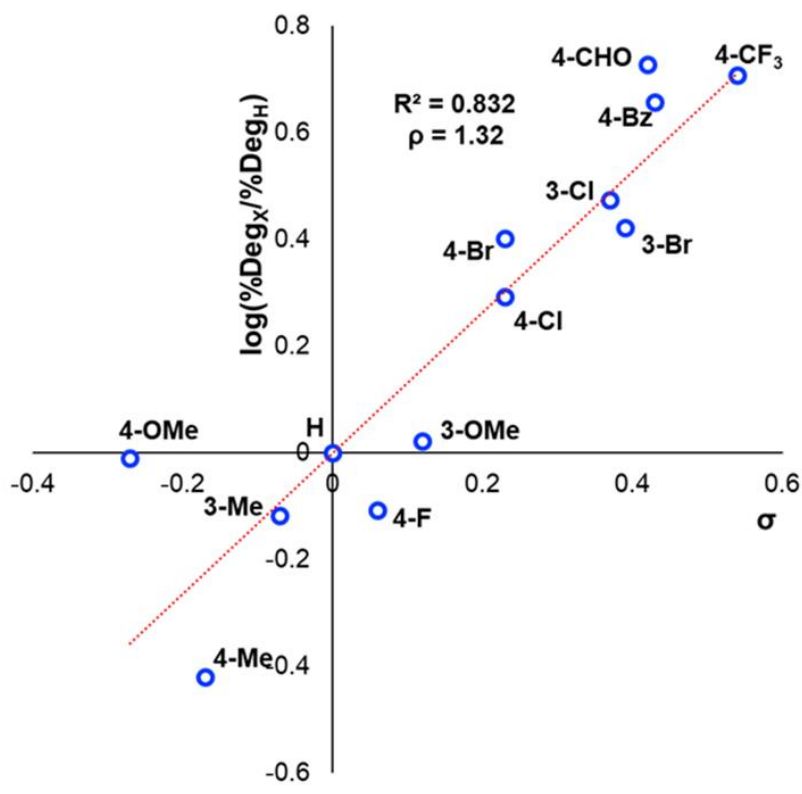

Figure 7. Hammett analysis of solution-phase degradation experiments shown in Figure 5, supporting the approximation that more electron-deficient ring substituents accelerate anilinium iodide degradation by demethylation as the predominant pathway.

anilinium cation degradation in the presence of TBACl versus $\mathrm{LiCl}$ and $\mathrm{KCl}$ is tentatively attributed to higher solubility of the former in DMSO. The low solubility of LiF, $\mathrm{Nal}, \mathrm{NaBr}, \mathrm{NaCl}$, and $\mathrm{KCl}$ in DMSO means it also possible that there was a lower-thancalculated (or even negligible) concentration of additive halide ions in solution. Indeed, with these reactions being conducted in NMR tubes, inefficient mixing may only exacerbate any solubility issues. Addition of TBAI (i.e. additional iodide beyond that in the anilinium salt) proved statistically similar to no additive at all. A related report on fluoride-mediated anilinium degradation has evidenced strong ion-pairing in TBAF that undergoes immediate salt metathesis in the presence of anilinium triflates to afford anilinium fluorides. ${ }^{12,58}$ If ion-pairing indeed dictates preorganisation prior to anilinium degradation, data in Figure 9 suggest that only the latter three additives contribute significant salt metathesis to form in situ ion pairs more prone to degradation than the parent anilinium iodide, $\mathbf{1 a}$.

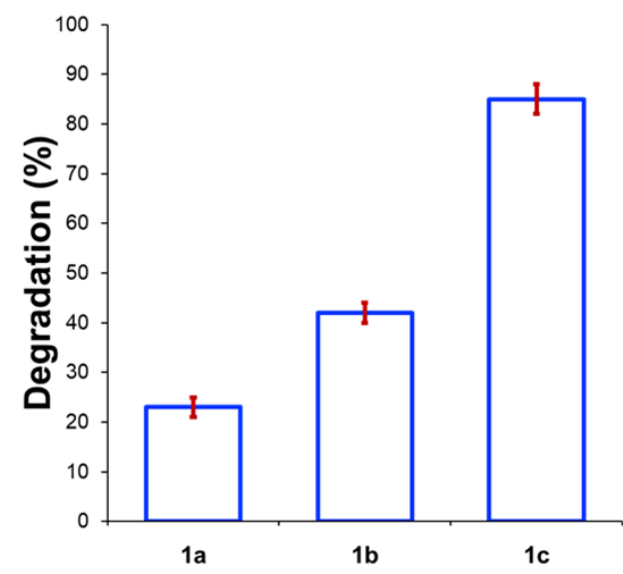

Figure 8. Degradation of $\mathbf{1 a}(X=\mathrm{I}), \mathbf{1} \mathbf{b}(X=\mathrm{Br})$, and $\mathbf{1} \mathbf{c}(X=\mathrm{Cl})$ upon heating in DMSO-d $(0.1 \mathrm{M})$ at $120^{\circ} \mathrm{C}$ for $20 \mathrm{~min}$. Results and errors are taken from triplicate runs.

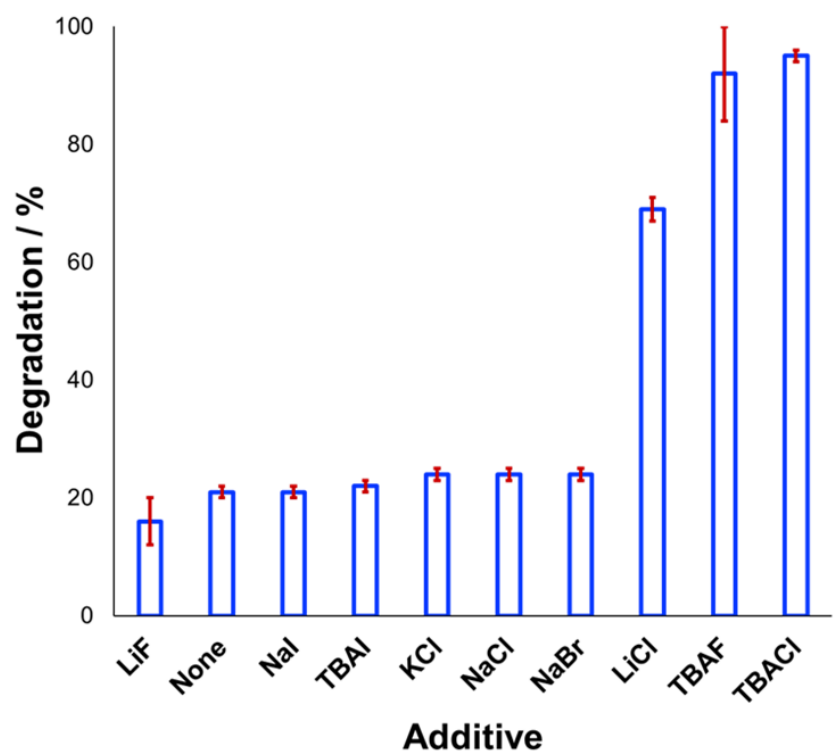

Figure 9. Relative degradation of $N, N, N$-trimethylanilinium iodide 1a upon heating in DMSO- $d_{6}(0.1 \mathrm{M})$ at $120^{\circ} \mathrm{C}$ for $20 \mathrm{~min}$ in the presence of 1 equiv. halide additive. Results and associated error bars taken from triplicate runs.

Another contributing factor in the apparent banality of LiF comes from its notably hygroscopic nature. In turn, this may have led to the introduction of water into the degradation mixture. A follow-up investigation showed that increasing water concentration - in the presence and absence of TBAF attenuated anilinium degradation (Figure 10), likely due to a decrease in halide nucleophilicity through more efficient anion solvation in water versus DMSO. Alternatively, the apparent decrease in halide nucleophilicity could also emerge through changes in the equilibrium, caused by enhanced solvation of ionic reactants as opposed to electronically neutral methyl

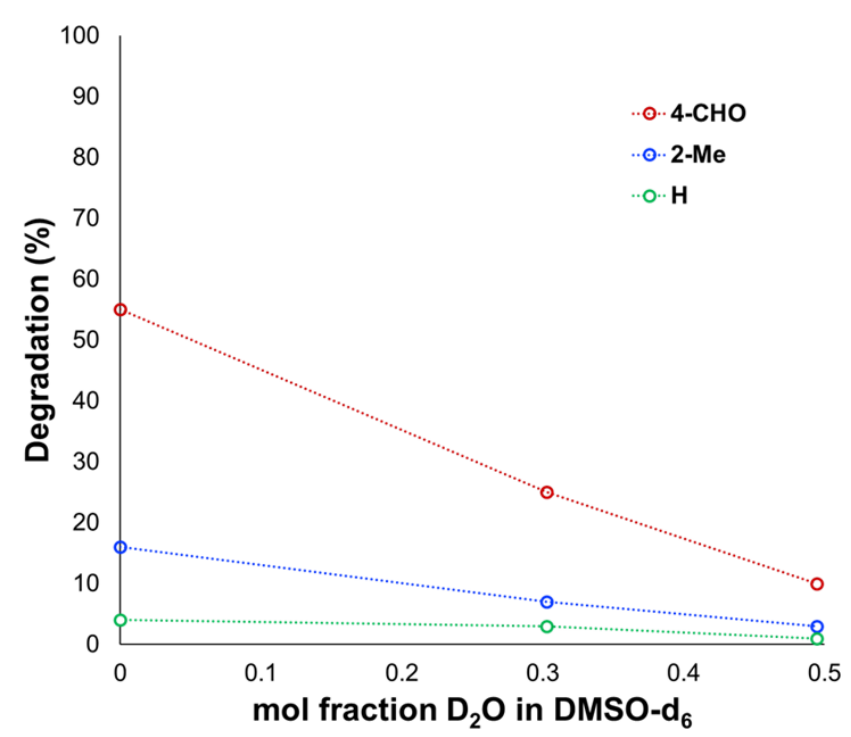

Figure 10. Attenuated degradation of anilinium iodides in the presence of additive concentrations of water. $\mathrm{N}, \mathrm{N}, \mathrm{N}$-trimethylanilinium iodides $(0.1 \mathrm{M})$ heated in DMSO-d with 0,10 or $20 \mathrm{v} / \mathrm{v}^{2} \mathrm{D}_{2} \mathrm{O}$, at $90{ }^{\circ} \mathrm{C}$ for $120 \mathrm{~min}$; maleic acid was used as an internal standard to calculate the concentration of the anilinium salt before and after heating. 
halide and dimethylanilines. ${ }^{55,59}$

Whereas the increased decomposition of the anilinium salts to generate the parent aniline and methyl halide in situ may be favourable for use in methylation chemistries, minimising degradation is desired for use in cross-coupling reactions. ${ }^{8-11,31-}$ ${ }^{34}$ Thus, we expanded our degradation studies to include a range of substituted anilinium salts bearing non-nucleophilic anions, including several anilinium partner anions employed in crosscoupling methodologies.

The solution-phase thermal degradation of these non-halide anilinium salts are shown in Figure 11. Non-nucleophilic counterions significantly retarded the decomposition of anilinium salts in solution, particularly when using tetrakis(3,5bis(trifluoromethyl)phenyl)borate (BArF-) salts which proved even more thermally stable than the commonly-applied triflate salts.

\section{NMR Degradation Kinetics}

We next sought to monitor the anilinium iodide degradation reaction time course to gain a richer insight into the active mechanism(s) of degradation. From a practical perspective, we hypothesised that this additional insight could enable practitioners to use anilinium salts as a controlled source of methyl iodide generated in situ. Again, ${ }^{1} \mathrm{H}$ NMR spectroscopy was employed to quantify reaction data. 4-formyl- $N, N, N$ trimethylanilinium iodide (3a), was selected as an appropriate salt to exemplify reaction kinetics since its degradation was significantly advanced (approximately three half-lives over 16 hours) to capture relevant mechanistic information in a feasible timescale. Additionally, the formyl proton on 3a was easily identifiable in NMR spectra due its isolated downfield chemical shift. The concentration of various species could be calculated against the internal standard (Figure 12).

The depletion of $\mathbf{3 a}$ could be monitored over time by following either the formyl, $-\mathrm{NMe}_{3}{ }^{+}$, or $o$-aryl proton. Similarly, the evolution of 4-formyl- $N, N$-dimethylaniline could be seen by following the analogous $-\mathrm{NMe}_{2}$, formyl and $o$-aryl protons.

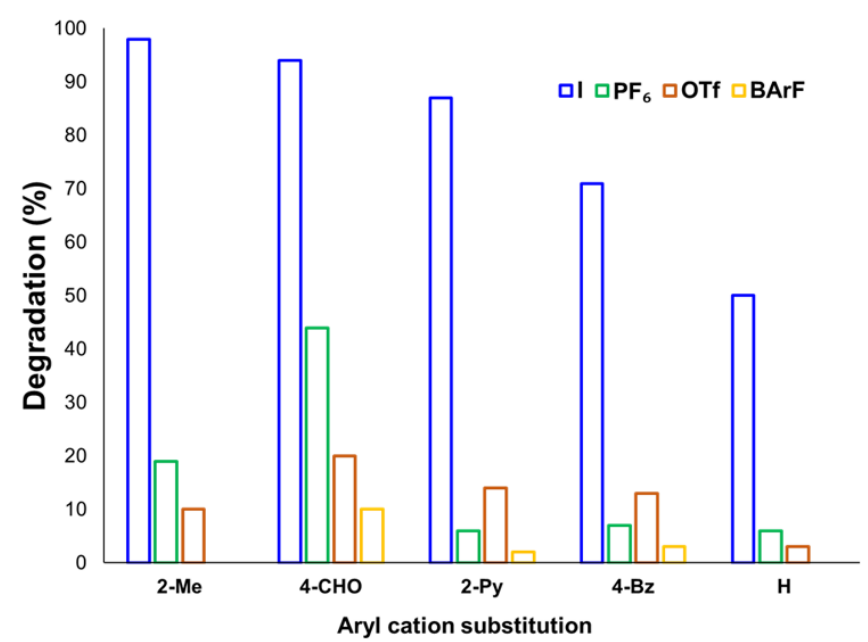

Figure 11. Relative degradation of $\mathrm{N}, \mathrm{N}, \mathrm{N}$-trimethylanilinium cations partnered with weakly coordinating (less nucleophilic) anions upon heating in DMSO- $\mathrm{d}_{6}(0.1 \mathrm{M})$ at 120 ${ }^{\circ} \mathrm{C}$ for $20 \mathrm{~min}$

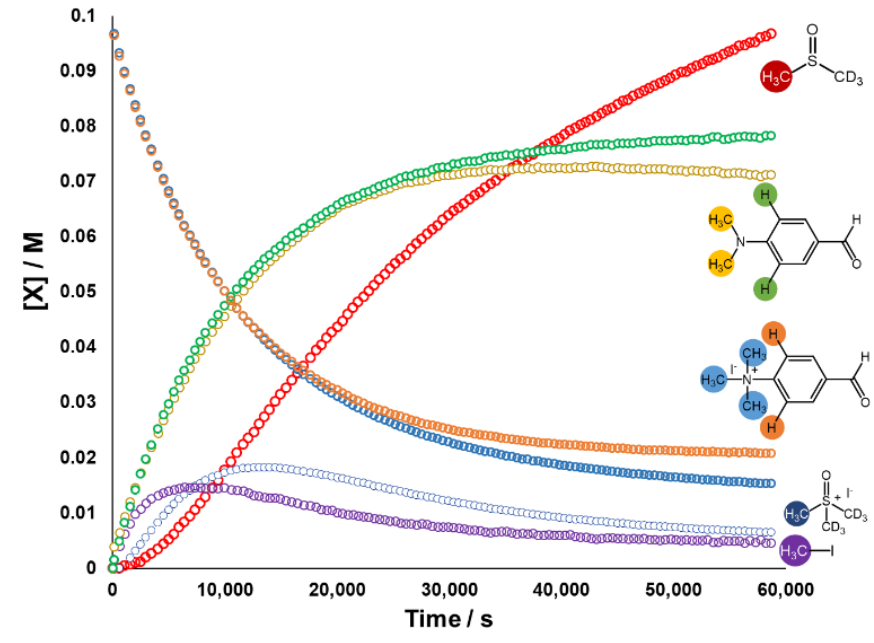

Figure 12. ${ }^{1} \mathrm{H}$ NMR time course for the degradation of 3a. Conditions: 3a (0.1 M) in DMSO- $\mathrm{d}_{6}(0.6 \mathrm{~mL}), 1,2,4,5$-tetramethylbenzene as internal standard $(0.06 \mathrm{M}$; not plotted) at $80^{\circ} \mathrm{C}$. Lines are colour-coded according to the proton shift followed, as indicated on the figure. Methyl transfer and isotopic scrambling were trackable.

Furthermore, the evolution of methyl iodide $\left(d_{0}\right)$ could be observed in the same experiment. Interestingly, the calculated concentration of both the anilinium or aniline via aromatic and formyl protons, whilst being equal, were each greater than the concentration calculated via the $N$-methyl groups (orange vs blue, and green vs yellow lines in Figure 12). This is suggestive of isotopic scrambling of $\mathrm{N}-\mathrm{CH}_{3}$ for $\mathrm{N}-\mathrm{CD}_{3}$ groups via interaction with the evidently non-innocent DMSO- $\mathrm{d}_{6}$ solvent. Deuterium scrambling is consistent with the emergence of trimethyl sulfoxonium $\left(\mathrm{d}_{6}, \mathrm{~h}_{3}\right)$ as a key intermediate, and DMSO$\mathrm{d}_{3}$ as a final product.

Our combined mechanistic evidence to this stage led to the tentative proposal of the mechanistic pathways summarised in Figure 13. Mass-calibrated diffusion-ordered NMR spectroscopy (DOSY) analysis of various anilinium salts in DMSO at room temperature did not support the existence of observable concentrations of higher order salt aggregates (see SI Section 13), though this does not directly rule out their transient existence. We proposed that the primary pathway via a relatively rapid approach to equilibrium of an approximately thermoneutral self-immolative degradation led by nucleophilic attack of iodide onto a $\mathrm{N}$-methyl unit of the anilinium cation, leading directly to the parent dimethylaniline and methyl iodide. In the bulk solvent, we proposed that DMSO could act as a S-centred nucleophile ${ }^{60,61}$ and attack the methyl iodide in an $\mathrm{S}_{\mathrm{N}} 2$ fashion, producing trimethylsulfoxonium iodide (TMSO$d_{6}, h_{3}$ iodide). The TMSO- $d_{6}, h_{3}$ cation could then undergo the reverse reaction with the aniline or instead undergo a selfimmolative process to release $d_{3}$-methyl iodide. The incorporation of $\mathrm{CD}_{3}$ in the anilinium cation, which could remain present in the degradative aniline product, is consistent with the difference in anilinium and aniline concentrations calculated using ${ }^{1} \mathrm{H}$ NMR spectroscopy, assuming negligible aromatic $H / D$ exchange. In further support of this hypothesis, 


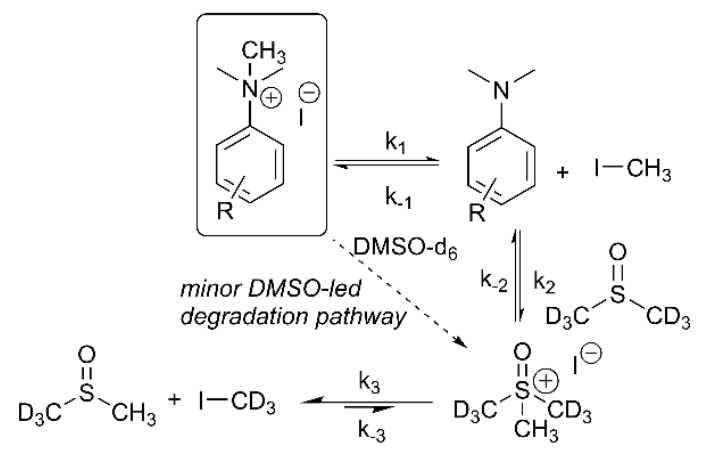

Figure 13. First proposed degradation pathway of $\mathrm{N}, \mathrm{N}, \mathrm{N}$-trimethylanilinium iodide salts in DMSO- $\mathrm{d}_{6}$, with rate constants assigned to each step. Minor deuterium scrambling steps have been omitted for clarity.

methyl iodide formed at an initial rate consistent with aniline formation (Figure 12 green and purple), whilst the TMSO- $d_{6}, h_{3}$ intermediate and, in turn, DMSO- $d_{3}$ product formation displayed an induction period. This suggested that iodide-led degradation of anilinium cation is the major (though not exclusive) precursor to methyl transfer to solvent.

The proposed degradation pathway takes place via a series of two-electron closed-shell processes. In order to probe for the possibility of radical degradation pathways, the degradation of 3a ( $p$-CHO) was carried out separately under UV light and in darkness. Comparatively, the amount of $\mathbf{3 a}$ degraded in each case was almost identical, suggesting that photo-degradation mechanisms are not dominant (see SI Section 15). The absence of radical-mediated mechanisms is also supported by additional control experiments in applied methylation chemistries (see Applications section below). It is plausible that the depletion of the anilinium salt could also occur through an $\mathrm{S}_{N} A$-type displacement of trimethylamine by the iodide counterion, however the corresponding aryl iodide was not observed (see SI Section 14).

The same ${ }^{1} \mathrm{H}$ NMR kinetics method was used to follow the degradation time course of a wider range of trimethylanilinium salts (SI Section 11). With the exception of 2a (2-py) and 14a (o$\mathrm{Me})$, most salts appeared to reach equilibrium that did not involve full degradation, consistent with a process that is approximately thermoneutral. Exploratory Swain-Lupton analysis ${ }^{62}$ was applied to solution-phase initial rate data to delineate field and resonance contributions of aryl ring substituents to salt degradation. This analysis revealed near equal field $(F)$ and resonance $(R)$ contributions $(52 \% R$ versus $48 \%$ R; Figure 14). The positive reaction constant ( $\rho=+3.71$ ) was consistent with earlier observations that more electrondeficient anilinium salts degrade more quickly (cf. Figures $\mathbf{5}$ and 7). Additional Swain-Lupton analysis using the simulated $k_{1}$ values based on the tentative reaction model shown in Figure 13 lead to similar conclusions ( $51 \%$ R versus $49 \% \mathrm{~F} ; \rho=+0.89$; see SI Section 11).

An important consideration when using a new reagent is the concentration at which it is to be used. Our time course experiments up until this point had been carried out with an anilinium salt concentration of $0.1 \mathrm{M}$ in $\mathrm{DMSO}_{-} \mathrm{d}_{6}$ for comparability. To understand the dependence of anilinium

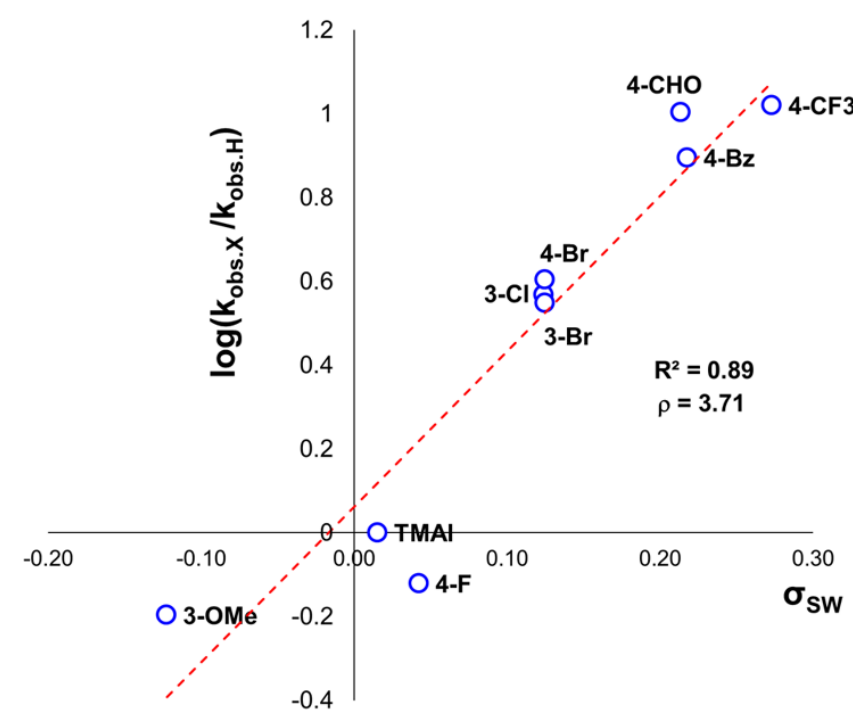

Figure 14. Swain-Lupton analysis using initial rate values (not explicitly derived rateconstants) for anilinium iodide degradation. Modified Hammett substituent constants were derived from Swain-Lupton $F$ and $R$ constants such that $\sigma s w=0.52 F+0.48 R$.

degradation on concentration, we carried out five additional degradation monitoring experiments at various anilinium iodide starting concentrations, [3a $]_{0}$. Inspection of the data revealed a deviation from linearity, providing evidence of greater than first order reaction order kinetics in [3a]o (Figure 15). This is not consistent with a simple self-immolation mechanism involving a single ion pair as the sole degradation mechanism, as suggested in Figure 13. Consistent with data reported in Figure 11, analysis of degradation of the BArF salt of [3a] via in situ NMR kinetics revealed that the background rate of degradation exclusively via the DMSO pathway (in the absence of nucleophilic iodide) was negligible at $80{ }^{\circ} \mathrm{C}$ (see SI Section 12). While DOSY NMR revealed no appreciable concentration of aggregates beyond the simple

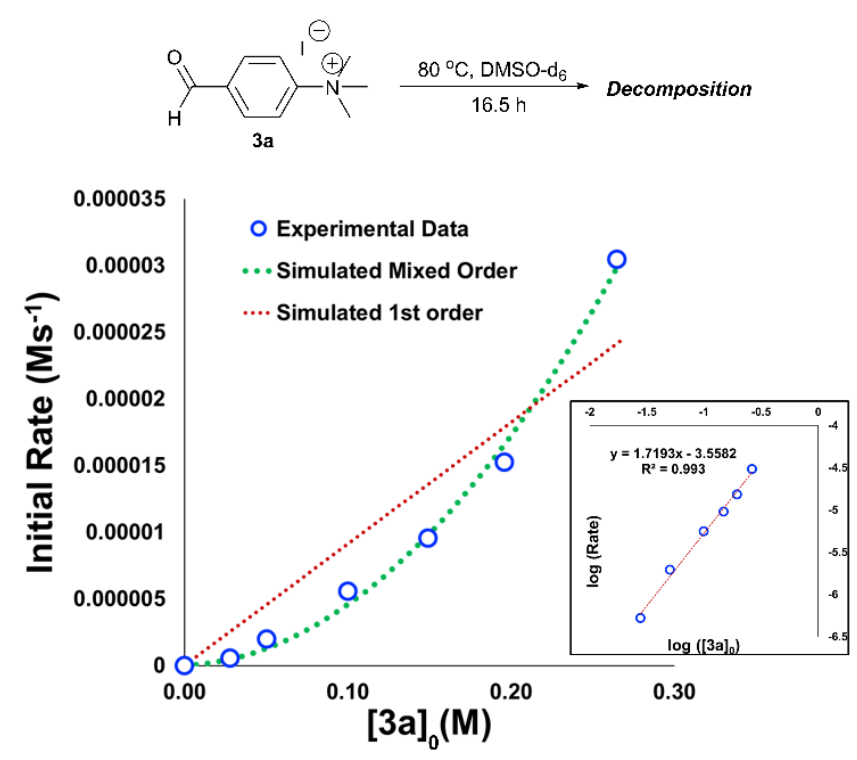

Figure 15. Main plot: Initial concentration of 3a versus calculated initial degradation rate evidencing mixed order kinetics. Real data (blue open circles) show agreement with simulated $1^{\text {st }}+2^{\text {nd }}$ mixed order kinetics (green dashed line) versus simulated first order 
kinetics (red dashed line). Inset: log-log plot showing apparent reaction order of approximately 1.7 in [3a].

anilinium iodide ion pair at room temperature under the range of concentrations studied, the presented data (most notably Figure 15) remain consistent with the possibility of degradation of the anilinium iodide salt via a transient dimeric ion pair. ${ }^{53}$ Dimeric degradation pathways were later considered computationally (below).

To estimate thermodynamic activation parameters, we conducted NMR monitoring studies between $50-80{ }^{\circ} \mathrm{C}$. Rate constants were estimated for a simplified first order iodide-led self-immolative degradation pathway at each temperature and an Eyring plot was produced using this data (Figure 16). The Eyring analysis revealed the activation enthalpy and entropy of the reaction as $\Delta \mathrm{H}^{\ddagger}=+127.0 \pm 4.0 \mathrm{kJmol}^{-1}$ and $\Delta \mathrm{S}^{\ddagger}=+34.8 \pm 11.7$ $\mathrm{Jmol}^{-1} \mathrm{~K}^{-1} . \Delta \mathrm{H}^{\ddagger}$ and $\Delta \mathrm{S}^{\ddagger}$ values for reactions involving changes in charge should be interpreted with caution as the values will include solvation dynamics and selection of appropriate rate constant from the mechanistic model. To cover this potential calculation error, we estimated Eyring parameters using both first and second order models, and in all circumstances, a positive entropy of activation was found. The positive activation entropy obtained in this case is hypothesised to be due to a reduced solvation demand as the charged reactants proceed to neutral products. ${ }^{63}$ This model is also consistent with reaction proceeding through ion pairs wherein the translational entropy penalty of bringing electrophile and nucleophile together has largely been paid. The overall $\Delta \mathrm{G}^{\ddagger}=+27.4 \mathrm{kcal} \mathrm{mol}^{-1}$ derived from these calculations is in good agreement with the related DFT-calculated barrier of $27.0 \mathrm{kcal} \mathrm{mol}^{-1}$ (see below).

To gain further insight into the degradation mechanism, a kinetic isotope effect study was conducted. A $d_{9}$-analogue (Ar$\left.\mathrm{N}\left(\mathrm{CD}_{3}\right)_{3}\right)$ of the $m$-bromo anilinium iodide salt (11a) was synthesised and subjected to an NMR degradation experiment. The rates of degradation determined by independent experiments. Whilst not conclusive on their own, all data were

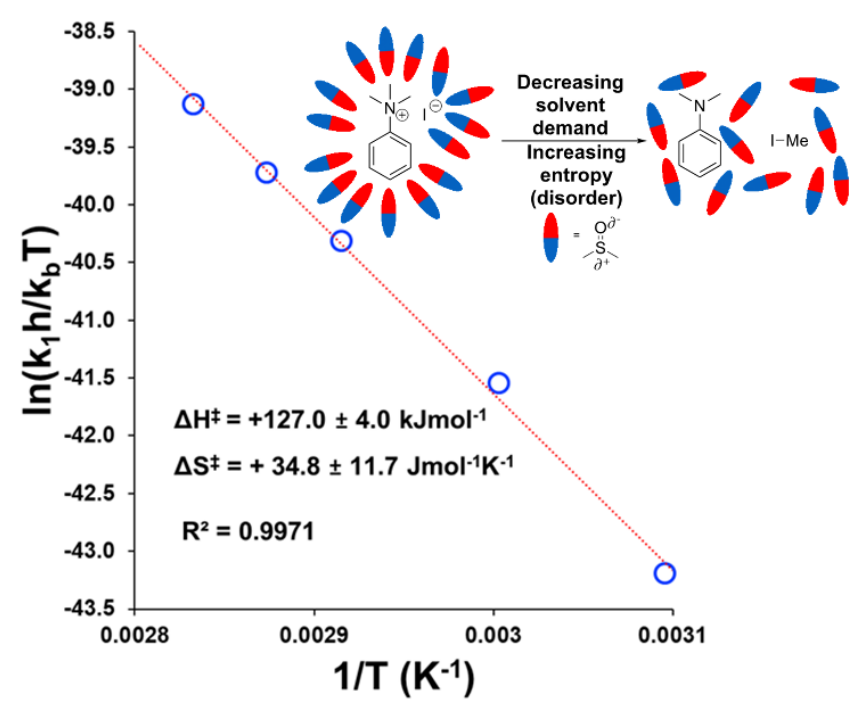

Figure 16. Exemplar Eyring plot for the degradation of 3 a between $50-80{ }^{\circ} \mathrm{C}$ showing counterintuitive positive entropy of activation. Inset: explanatory cartoon model citing reduced solvent demand as the root cause of the observed entropy value. consistent with there being no primary KIE (approx. 1.18), thus ruling out mechanisms involving direct deprotonation (i.e. ylide formation) on the anilinium cation N-Me groups (see SI Section 19). These data are also qualitatively consistent with DFTcalculated KIE range of 1.29-1.47 covering 4 major mechanistic hypotheses (see below).

\section{Applications of Mechanistic Analysis in Methylation Chemistries}

Applying our new mechanistic data, we examined the broader use of anilinium salts as methylating reagents. Degradation analysis revealed the range of thermal and additive stabilities (knowledge applicable to both cross coupling and process safety strategies). We hypothesised that those anilinium salts presenting as thermally unstable would, in turn, release methyl iodide in situ most readily, and also be more susceptible to direct nucleophilic attack, thus behaving as a viable methyl iodide replacement. For a comparative application in methylation, phenol O-alkylation was chosen as it remains one of the most used reactions in the pharmaceutical industry. 64,65 4-tBu-phenol (16) was selected as a suitable substrate for initial exploratory study. The conversion of 16 to4tBu-anisole (17) was calculated from the reaction mixture after $3 \mathrm{~h}$ via ${ }^{1} \mathrm{H}$ NMR spectroscopy, and the average conversion of each salt from triplicate experiments is shown in Figure $\mathbf{1 7 .}$

From these experiments, the hypothesised trend of thermally unstable anilinium salts leading to more efficient methylation did not hold. Indeed, three of the most thermally unstable anilinium iodides $\mathbf{2 a - 4 a}$ and $\mathbf{1 5 a}$ - all carrying strong electron- withdrawing aryl substituents - led to measurable quantities of $S_{N} A r$ products (18-21) and poor yields of desired methylation products. Indeed, analysis of thermal degradation

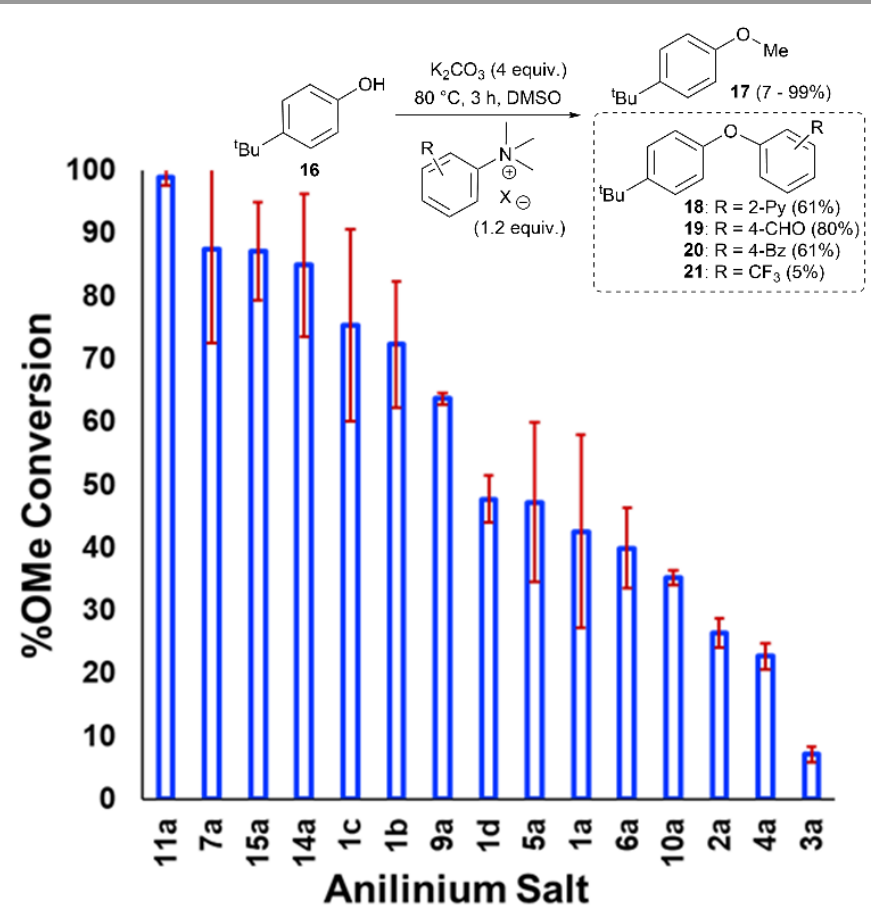

Figure 17. Screen of O-methylation capability enabled my anilinium salts. 
versus O-methylation efficiency within the anilinium salt library revealed that only those unstable salts bearing no resonance withdrawing aryl substituents served as efficient methylating reagents.

The anilinium iodide salt most reactive for methylation was shown to be 11a $(m-\mathrm{Br})$, giving near-quantitative conversion to 16 with minimal variance (Figure 18). We were surprised to see that salt 10a $(3-\mathrm{Cl})$, which has a similar degradative profile to 11a (Figures 5, 7, and 14), was considerably less reactive to methyl transfer under the applied conditions. More expectedly, 7a (4-Br) gave high conversions in comparison to the 5 a (4-F) and $6 \mathrm{a}(4-\mathrm{Cl})$ analogues, the latter two presenting as more stable to thermal degradation. 14a (2-Me), the only salt identified as prone to degradation due to steric bulk, gave an average of $85 \pm 11 \%$ conversion to the methylated product under the conditions tested. Salt $\mathbf{1 4 a}$ presents a viable alternative to 11a where methylation (or cross-coupling) reactions might be compromised by the presence of the $3-\mathrm{Br}$ substituent in 11a. Surprisingly, 2a (2-Py), 3a (4-CHO), and 4a (4-Bz) anilinium iodide salts, each gave a relatively low conversion to $\mathbf{1 7}$ despite being the most thermally unstable salts in solution-phase. On closer inspection, it was found that $S_{N} A r$ products 18,19 , and 20, were formed from use of the most resonance withdrawing anilinium iodide salts (Figure 17). The $S_{N} A r$ reactivity presumably arises from 4-tBu-phenoxide displacing trimethylamine from the respective anilinium salt. 6,56

The trend in methylation ability for the halide salt series $\mathbf{1 a}$ c correlated with extent of thermal degradation in solution, with the chloride salt giving higher conversions to methylated product than the analogous bromide and iodide salts. The triflate salt (1e) was comparative in methylating ability to the iodide salt (1a). Our mechanistic degradation studies showed that methyl iodide was produced upon heating the anilinium iodide salts in DMSO, raising the question of whether

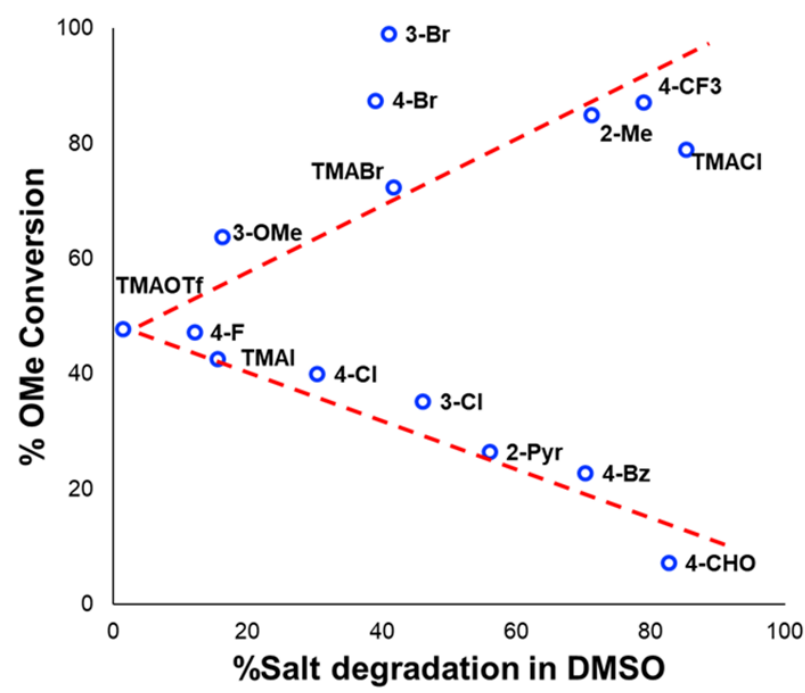

Figure 18. Partition of anilinium salts accordingly to their thermal degradation and Omethylation behaviours. Non-iodide salts are indicated where appropriate. The dotted lines are included solely as a guide for the eye. methylation of $\mathbf{1 6}$ occurs from reaction of the phenoxide nucleophile directly with the anilinium cation, from a methyl halide generated in situ, or both. The shared trend in anilinium degradation and phenol methylation reactivity $(\mathbf{1} \mathbf{c}>\mathbf{1} \mathbf{b}>\mathbf{1 a})$ suggests that decomposition to the methyl halide is important for reactivity. However, the comparable methylating ability of1a $(X=I)$ and 1e (X=OTf), despite the large difference in stability (see Figure $\mathbf{9}$ and Figure 13), indicates that attack directly onto the anilinium salt also occurs, assuming negligible degradation of the triflate salt to produce methyl triflate in situ (Figure 11). We propose that a combination of direct reaction with the anilinium salts and indirect reaction (via methyl iodide generation) in the phenol methylation both occur under the reaction conditions employed. This proposal is further elaborated in discussion below.

To compare the effectiveness of methylating reagent 11 a to common electrophilic methylating reagents, the methylation of phenol $\mathbf{1 6}$ was carried out using methyl iodide, methyl triflate, methyl tosylate, dimethylsulfate, and dimethylcarbonate under our chosen reaction conditions (Table $\mathbf{1}$ ).

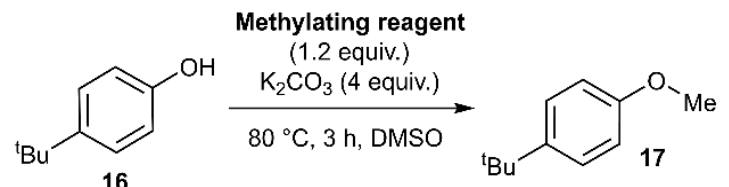

\begin{tabular}{|c|c|c|}
\hline Entry & Methylating Reagent & Yield (\%) \\
\hline 1 & 11a & 98 \\
\hline 2 & Mel & 79 \\
\hline 3 & MeOTf & 2 \\
\hline 4 & $\mathrm{MeOTs}_{2}$ & 62 \\
\hline 5 & $\mathrm{Me}_{2} \mathrm{SO}_{4}$ & 29 \\
\hline 6 & dimethyl carbonate & 0 \\
\hline 7 & dimethyl carbonate (+10 mol\% DBU) & 0 \\
\hline
\end{tabular}

Table 1. Comparative methylation with anilinium salt 11a versus common electrophilic methylating reagents.

We next investigated the ability of 11a to methylate a range of phenols and related nucleophiles under our applied reaction conditions (Figure 19, see Experimental Supporting Information Section 20 for optimisation). A range of substituents and structural complexities around the phenol ring were tolerated in these methylation reactions, and most with good to excellent yields (17, 22-31, 34). Additionally, thiophenol and benzoic acid could be methylated under the applied conditions to give thioether $\mathbf{3 2}$ and ester $\mathbf{3 3}$ with good $\mathbf{7 7 \%}$ and $\mathbf{8 0 \%}$ yields, respectively. Notably, having used the earlier mechanistic study to guide choice of reagent (Figure 17), no $S_{N} A r$ product was observed in any of our reactions using 11a as a methylating reagent. 14a (o-methyl) was explored as an alternative methylating reagent for cases where a $m-\mathrm{Br}$ substituent could cause undesired reactivity, and was shown to be effective in the formation of $17(86 \%), 31(62 \%)$ and $34(84 \%)$. Using the same subset of substrates, NMP was shown to be a viable alternative solvent, enabling the synthesis of 17, 31, and $\mathbf{3 4}$ in 96, 94, and $84 \%$ yield, respectively. 


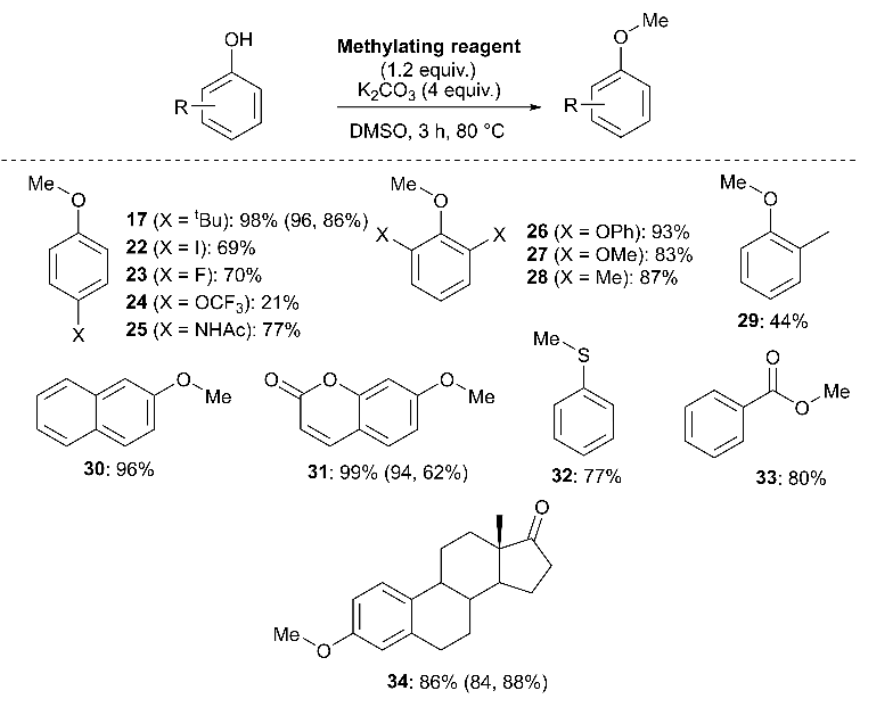

Figure 19. Methylation of phenols, thiophenol and benzoic acid using 11a. Yields in parentheses obtained with $14 \mathrm{a}(\mathrm{o}-\mathrm{Me})$ as the methylating reagent (left number) or for 8 $h$ in NMP as solvent (right number), under otherwise identical conditions.

We also investigated whether or not 11a could provide any uniquely applicable regiochemical control when multiple nucleophilic phenol sites are present on a substrate. To probe this, 2',4'-dihydroxyacetophenone was subjected to methylation with 11a and methyl iodide under our standard conditions, showing exploitable differences in reactivity and regioselectivity (Figure 20). A deeper analysis of this synthetic application is the subject of future investigation.

\section{Experimental Mechanistic Analysis of Active Methylation Species in Solution Using Anilinium Salts}

Having demonstrated the potential of $N, N, N$ trimethylanilinium (primarily iodide) salts to act as competent

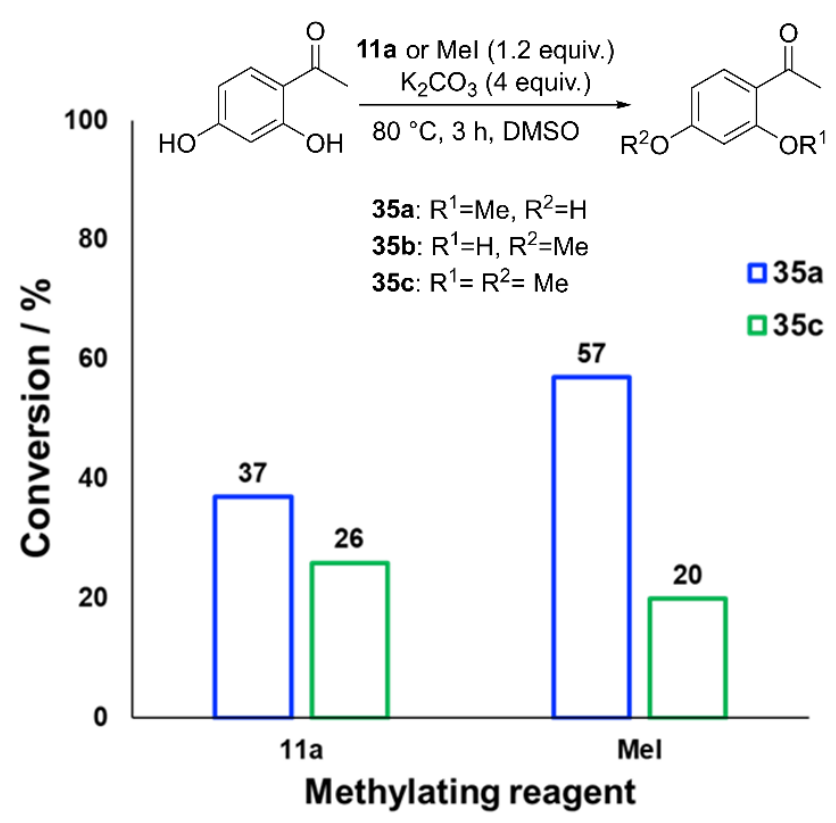

Figure 20. Conversion of $2^{\prime}, 4^{\prime}$-dihydroxyacetophenone to 35a-c using 11a or methy iodide, demonstrating the possibility of exploiting differing methylation product selectivities. In both cases, no $\mathbf{3 5 b}$ was formed. methylating reagents, we next aimed to understand the plausible mechanism(s) by which these O-methylation reactions occur. Our understanding of anilinium thermal degradation in DMSO- $d_{6}$ suggested multiple possible methylation pathways: Path A: from methyl iodide generated from TMSO-I degradation, Path B: from TMSO-I that forms in situ through methylation of the non-innocent DMSO solvent by methyl iodide, Path C: via methyl iodide generated from the anilinium iodide degradation, and Path D: direct attack of the nucleophile onto an $\mathrm{N}$-methyl group of the anilinium cation, (Figure 21).

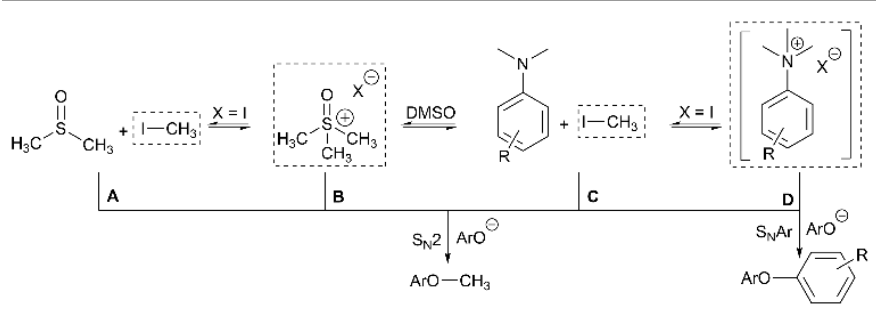

Figure 21. Sources of electrophilic methylation reagent when employing an anilinium iodide in DMSO. The dotted boxes highlight the source of "Me" at each side of the connected equilibria. Competing $\mathrm{S}_{N} A r$ reactivity for the anilinium salt is also shown.

To address the question of whether or not substrate methylation comes solely from the anilinium degradation-led generation of methyl iodide (Path C), we carried out the methylation of $\mathbf{1 6}$ under our chosen (loosely-optimised) reaction conditions using $m$-bromo- $N, N, N$-trimethylanilnium $\mathrm{PF}_{6}$ (11d) and BArF (11f) salts (Table 2). In these reactions where methyl iodide could not be generated, 17 was formed in $45 \%$ (entry 2 ) and $50 \%$ (entry 3 ) yield, compared to $98 \%$ yield when using iodide salt 11a (entry 1). This showed that the generation of methyl iodide likely accompanies efficient methylation of phenoxide substrates, but is not a necessity for the reaction to occur under the chosen conditions. Given the knowledge that anilinium $\mathrm{PF}_{6}$ and BArF salts can partially decompose in DMSO$\mathrm{d}_{6}$ to give the TMSO cation (see Figure 11), it is possible that methylation could occur in these reactions from the anilinium directly (Path D) or the TMSO cation itself (Path B).

\begin{tabular}{|c|c|c|c|c|c|}
\hline Entry & $\begin{array}{c}\text { Methylating } \\
\text { Reagent }\end{array}$ & Anion & Solvent & $\begin{array}{r}\text { Additive } \\
\text { (equiv.) }\end{array}$ & $\begin{array}{c}\text { O- } \\
\text { methylation } \\
\text { Yield (\%) } \\
\end{array}$ \\
\hline 1 & 11a & I & DMSO & - & 98 \\
\hline 2 & $11 \mathrm{~d}$ & $\mathrm{PF}_{6}$ & DMSO & - & 45 \\
\hline 3 & $11 \mathrm{f}$ & BArF & DMSO & - & 50 \\
\hline 4 & TMSO-I & 1 & DMSO & - & 76 \\
\hline 5 & $\mathrm{TMSO}^{\mathrm{P} \mathrm{PF}_{6}}$ & $\mathrm{PF}_{6}$ & DMSO & - & 57 \\
\hline 6 & TMSO-BArF & BArF & DMSO & - & 53 \\
\hline 7 & $11 a$ & 1 & NMP & - & 88 \\
\hline 8 & 11d & $\mathrm{PF}_{6}$ & NMP & - & 11 \\
\hline 9 & 11a & 1 & DMSO & TEMPO (3) & 65 \\
\hline 10 & $11 a$ & I & DMSO & (1.2) & $\begin{array}{c}86 \text { (no } \\
\text { consumption } \\
\text { of additive) }\end{array}$ \\
\hline
\end{tabular}

Table 2. Mechanistic experiments investigating the conversion of $\mathbf{1 6}$ to $\mathbf{1 7}$ with various methylating reagents in DMSO and NMP. Conditions: 16 ( 1 mmol, 1 equiv.), methylating reagent (1.2 equiv.), $\mathrm{K}_{2} \mathrm{CO}_{3}$ (4 equiv.), additive (various equiv.), solvent $(2 \mathrm{~mL}), 80^{\circ} \mathrm{C}, 3 \mathrm{~h}$. 
To investigate this further, we ran the same methylation reactions with TMSO salts in the place of anilinium salts. TMSOI formed $\mathbf{1 7}$ in $\mathbf{7 6 \%}$ yield (entry 4). We were able to demonstrate through NMR analysis that TMSOI decomposes thermally to give DMSO and methyl iodide at $80^{\circ} \mathrm{C}$, the reverse reaction was also shown to occur (SI Section 22). This finding raised the question as to whether $\mathrm{TMSO}^{+}$acts directly as a source of methyl group (Path B), or if the methylation is simply occurring from methyl iodide produced via its thermal decomposition (Path A). TMSO-PF 6 and TMSO-BArF were synthesised and subsequently used in methylation reactions to afford the anisole 17 in 57\% (entry 5) and 53\% yield (entry 6), respectively. Whilst this suggests that the $\mathrm{TMSO}^{+}$cation is capable of methylating phenols, it does not rule out the possibility that nucleophiles can directly attack the anilinium cation to form the methylated product (Path D). To investigate the ability of the anilinium cation to directly methylate the substrate, the ability to generate $\mathrm{TMSO}^{+}$in the reaction mixture would also have to be removed. Accordingly, we ran the methylation reactions in $\mathrm{N}$-methyl-2-pyrolidone (NMP). Anilinium iodide 11a was used to methylate 16 , giving $88 \%$ of the methylated product (entry 7 ).

We then repeated the reaction using anilinium $\mathrm{PF}_{6}$ salt $\mathbf{1 1 d}$ as the methylating reagent, which gave only $11 \%$ yield of $\mathbf{1 7}$, strongly suggesting that the degradation of the anilinium iodide to release methyl iodide in situ is a key contributor to the reaction (Path C); computational studies reveal that the barriers for degradation and direct reaction are close such that reaction flux through each pathway likely depends on the details of concentration, temperature and the identity of the anilinium salt. Furthermore, additive experiments (entries 9 and 10) probing the presence of radical-centred degradation and methylation mechanisms were carried out. These data remained consistent with experimental and theoretical evidence that anilinium iodide degradation was primarily via closed pathway, and that the principle self-immolative degradation pathway was faster than degradation via DMSO participation (see Figures 9, 11, and 12).

\section{Computational Analysis of Anilinium Salt Degradation and Methylation Mechanisms}

Further mechanistic insight for both anilinium salt degradation and methylation was sought from computational studies. The computations were all performed at the M06-2X/6$31+G^{* *}$ level of theory and with IEF-PCM for DMSO as the solvation model, all performed in Gaussian09.66 M06-2X was designed to be a preferred method for the investigation of reaction barriers, and so is appropriate for this task. Vibrational and standard state corrections were implemented using Goodvibes (with concentration values $1 \mathrm{M}$ and $14.1 \mathrm{M}$ respectively for solutes and DMSO; a frequency cut-off of 100 $\mathrm{cm}^{-1}$ was used). 67

A key first step was to establish the likely speciation of the anilinium salts, as either separated ions and/or as ion pairs. The latter was supported by our DOSY NMR measurements (SI Section 13). These calculations are thus particularly sensitive to the applied solvent model. Two geometries were considered for the ion-pair, one with the halide placed as an extension of the ipso- $\mathrm{C}_{\text {aromatic }}-\mathrm{N}$ bond, and the other with the halide between the $\mathrm{N}$-methyl groups and the ortho $\mathrm{C}-\mathrm{H}$ position. The latter was found to be energetically preferred in all cases investigated (see Computational Supporting Information Section 2). Calculations at $353 \mathrm{~K}\left(80^{\circ} \mathrm{C}\right)$ predicted all of the iodide salts to have a small (0.8-3.8 kcal mol-1) preference to be ion-paired. The bromides are computed to have a mix of preferences, with 4-CHO and 3$\mathrm{Br}$ showing $0.5 \mathrm{kcal} \mathrm{mol}^{-1}$ preferences to be ion pairs, whilst 2Me and 2-pyridyl show preferences to be separate ions. Chloride salts were computed to all have an energetic preference (of 0.2 to $2.8 \mathrm{kcal} / \mathrm{mol}$ ) to be separated ions apart from for $3-\mathrm{Cl}$ and $3-\mathrm{Br}$.

Reaction profiles were computed for each of the anilinium salts in which both degradative reactions (Mel formation and reaction with solvent) and productive reactions ( $\mathrm{S}_{\mathrm{N}} 2$ and $\mathrm{S}_{\mathrm{N}} \mathrm{Ar}$ with phenolate) are compared. An example is given for $\mathbf{3 a}$ in Figure 22 where:

State $\mathbf{A}=$ the reactant anilinium salt (as an ion-pair)

State $\mathbf{B}=$ methyl transfer to solvent DMSO

State $\mathbf{C}=$ self-immolative methyl transfer to halide (i.e. Mel is formed)

State $D=S_{N} 2$ methylation of phenolate, and

State $\mathbf{E}=\mathrm{S}_{\mathrm{N}} \mathrm{Ar}$ reaction.

Some features are consistent for each of the iodide salts. First, the reaction of the anilinium iodide with DMSO is kinetically and thermodynamically disfavoured (Figure 22, A$>B$ ). The formation of Mel (Figure 22, A->C) is close to thermoneutral and has a barrier that is readily accessible at the elevated temperatures employed in the experimental studies. Consequently, the reverse reaction in which anilinium reforms is also accessible. The barrier for methyl iodide to react with DMSO is very close to that for methyl iodide to react with aniline such that any methyl iodide formed could be expected to partition between formation of anilinium and of sulfoxonium. These calculations are consistent with the observed delay in sulfoxonium formation coming only after methyl iodide formation, the intermediate formation both methyl iodide and sulfoxonium, the induction period in the observed formation of DMSO- $d_{3}$, and the observed positions of H/D scrambling (Figure 12).

The reactions with phenolate, $\mathrm{S}_{\mathrm{N}} 2$ (Figure 22, $\mathrm{C}->\mathrm{D}$ ) and $\mathrm{S}_{\mathrm{N}} \mathrm{Ar}$ (Figure 22, $A->E$ ) have barriers that are low and largely irreversible. For salts 2a (2-Pyr), 3a (4-CHO), and 4-Bz (4a), the barrier for $S_{N} A r$ is computed to be the most lowered versus $S_{N} 2$ (Figure 23), qualitatively corresponding to the observation of $\mathrm{S}_{N} \mathrm{Ar}$ product dominating the product mixture (Figure 18). More electron-donating substituents are computed to have a preference for $\mathrm{S}_{\mathrm{N}} 2$ but this is accompanied by an overall higher barrier for reaction; thus, iodide salts predicted to be selective for methylation over arylation are also predicted to react more slowly. Although the 4-CHO salt (3a) is expected to have barriers for direct reaction of phenolate with salt that are significantly lower than those for formation of Mel, this is not the case for all anilinium salts universally. For instance, the unsubstituted iodide salt, 1a (Computational Supporting Information Section 


\section{ARTICLE}

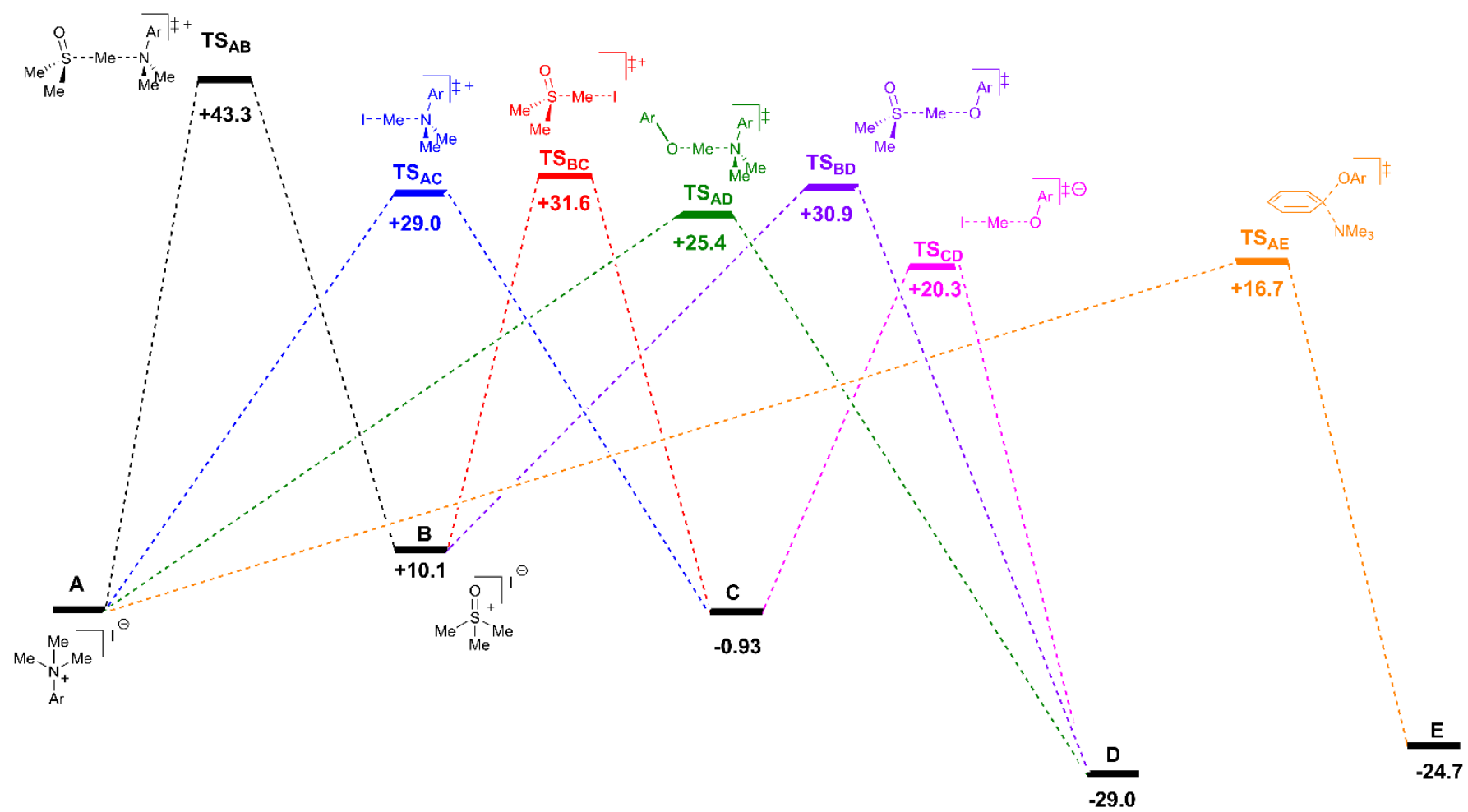

Figure 22. DFT-derived potential energy surface, summarising experimentally-consistent mechanisms for anilinium iodide degradation and anilinium-mediated O-methylation.

3), is computed to have a barrier to formation of Mel of 29.9 $\mathrm{kcal} / \mathrm{mol}$, a barrier to $\mathrm{S}_{\mathrm{N}} A$ r of $29.8 \mathrm{kcal} / \mathrm{mol}$, and a barrier to $\mathrm{S}_{\mathrm{N}} 2$ with phenoxide of $28.8 \mathrm{kcal} / \mathrm{mol}$, and thus all three processes might be expected to be operating experimentally. Whilst the calculations are not able to explicitly corroborate 11a (3-Br) as the experimentally optimum methylating reagent, they are suggestive of this salt as having a good balance between the likely degree of degradation and the selectivity between $S_{N} 2$ and $S_{N} A r$ reactions, thus providing an effective and safer 'slow bleed' alternative to methyl iodide.

Figure $\mathbf{2 4}$ shows three alternative modes of anilinium iodide self-immolation in ion-pairs, beyond simple first order selfreaction, that were considered computationally. Of all 4 mechanisms, $\mathrm{A}$ and $\mathrm{C}$ were consistently the most accessible across calculations involving salts $1 \mathrm{a}, 3 \mathrm{a}$, and $11 \mathrm{a}$; thus, at high concentrations, second order contributions to anilinium iodide degradation. This is consistent with the observed deviation from linear concentration dependence reported in Figure $\mathbf{1 5 .}$

\section{Limitations of Mechanistic Analysis}

Our mechanistic analysis - both experimental and computational - is consistent with at least two operative mech-

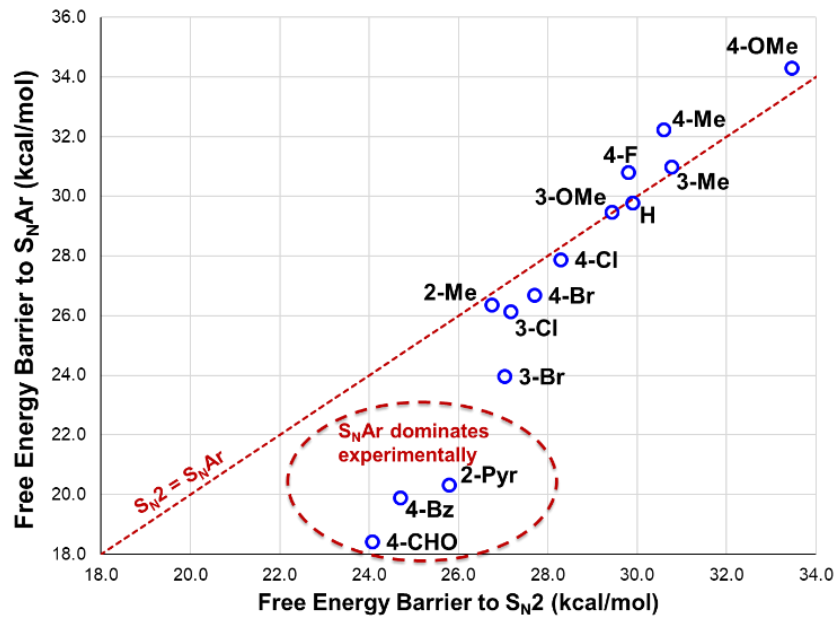

Figure 23. DFT-computed comparison of SNAr and SN2 barriers for reaction of phenolate with anilinium cation. Method: M06-2X/6-31+G**/IEFPCM (DMSO). $\mathrm{T}=353.15 \mathrm{~K}\left(80^{\circ} \mathrm{C}\right)$.

anisms of anilinium iodide degradation. The first and most dominant is simple first order self-immolation; one ion-pair degrades to one molecule of dimethylaniline and one molecule 


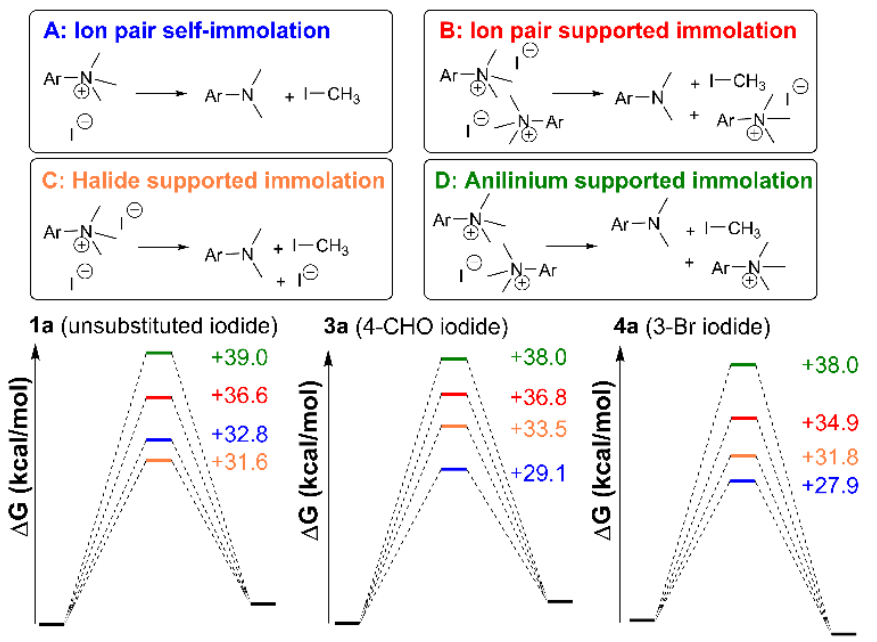

Figure 24. Four anilinium salt degradation mechanisms for which DFT-computed solutions were found.

of methyl iodide. The second mechanism, accessible at higher concentrations of anilinium iodide, is proposed to be (at least in part) second order with respect to the anilinium iodide ion pair. Further microkinetic analysis remains consistent with both first and second order processes being operative (see Experimental SI Section 17). The main unavoidable assumption in all mechanistic analysis, is that these reactions proceed in an unchanging medium that presents unchanging physical properties as the main reactions proceed. In reality, an unchanging medium is unlikely, especially for such reactions proceeding from charged reactants to neutral products. Conductivity measurements (Figure 25, and Experimental SI Section 25) and scans of solvent model dielectric constant versus $\Delta G$ (Computational Supporting Information) show that the physical properties of the reaction medium change with time.

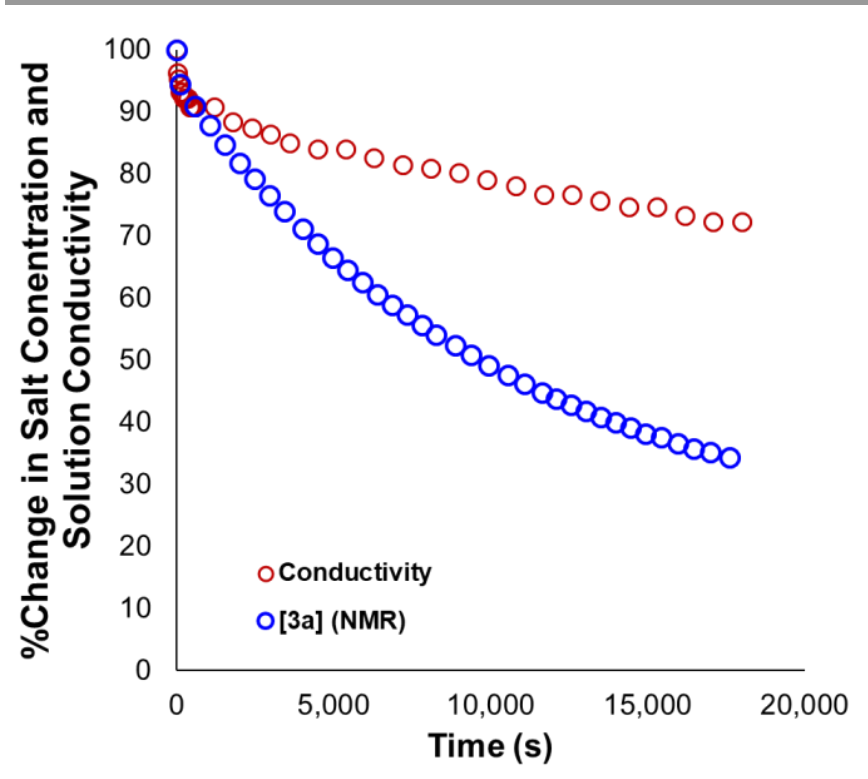

Figure 25. Relative change in measured solution conductivity relative to changes in $\mathbf{3 a}$ salt concentration during degradation. Conditions: $3 a(0.1 \mathrm{M})$ in $\mathrm{DMSO}^{-\mathrm{d}_{6}}(0.6 \mathrm{~mL})$, 1,2,4,5-tetramethylbenzene as internal standard $\left(0.06 \mathrm{M}\right.$; not plotted) at $80^{\circ} \mathrm{C}$.

\section{Conclusions}

We have presented solid and solution phase thermal degradation analyses, kinetic studies, isotopic labelling, and computational modelling showing that $\mathrm{N}, \mathrm{N}, \mathrm{N}$ trimethylanilinium salts are likely to degrade in a selfdestructive process. Taken together, the mechanistic experiments forced a partial revision of our initial mechanistic hypothesis from Figure 13, as displayed in Figure 26. Increasing the anilinium counter-anion nucleophilicity, solution concentration, electron-withdrawing aryl substituent power, soluble non-iodide halides, and solution temperature were all found to increase the rate of salt degradation. The minimal impact of solvent-led degradation pathways was supported by computational modelling of the two degradation pathways. A range of $\mathrm{N}, \mathrm{N}, \mathrm{N}$-trimethylanilinium salts was tested as $\mathrm{O}$ methylation reagents, with aryl-substitution and counterion species shown to affect methylating ability. 3-Br- $N, N, N-$ trimethylanilinium iodide (11a) was shown to be an effective methylating reagent for a variety of phenols in DMSO and NMP. Mechanistic studies suggest that in situ generated methyl iodide is likely to be the main methylating reagent in these reactions. From a practical standpoint, we thus propose that carefully selected anilinium iodide salt could function as a safer, crystalline, and storeable 'slow bleed' alternative to using methyl iodide directly. Moreover, the same studies highlighted those salts for which concerted $S_{N} A r$ at the ipso carbon of the parent anilinium salts presents dominant reactivity, a key consideration for chemists aiming to exploit these same salts for cross-coupling as opposed to methylation reactivity.

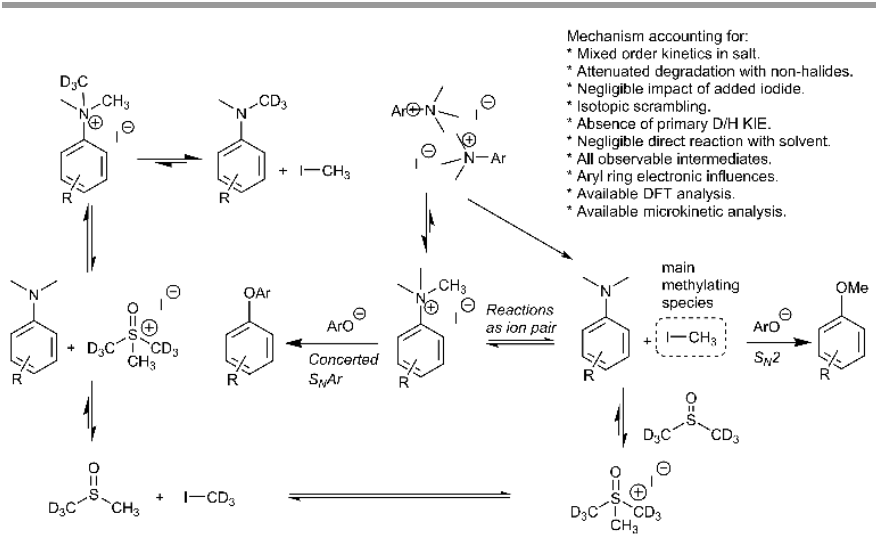

Figure 26. Revised mechanistic model for anilinium iodide degradation, consistent with all available experimental and computational mechanistic evidence.

\section{Conflicts of interest}

There are no conflicts to declare.

\section{Acknowledgements}

MR thanks the Leverhulme Trust for fellowship funding (ECF2016-264). JBW thanks GlaxoSmithKline for funding. MR, DM, $\mathrm{CY}$, and JBW thank Drs John Liggat and Len Berlouis for helpful discussions during the course of solid-state thermal degradation 
investigations. MR and JBW thank the EPSRC for funding via Prosperity partnership EP/S035990/1.

\section{Notes and references}

1 S. E. Denmark, N. D. Gould and L. M. Wolf, J. Org. Chem., 2011, 76, 4260-4336.

2 H. J. Davis, M. T. Mihai and R. J. Phipps, J. Am. Chem. Soc., 2016, 138, 12759-12762.

3 K. Brak and E. N. Jacobsen, Angew. Chemie Int. Ed., 2013, 52, 534-561. N. A. Shamsutdinova, I. D. Strelnik, E. I. Musina, T. P. Gerasimova, S. A. Katsyuba, V. M. Babaev, D. B. Krivolapov, I. A. Litvinov, A. R. Mustafina, A. A. Karasik and O. G. Sinyashin, New J. Chem., 2016, 40, 9853-9861. P. H. J. Carlsen, K. Liberkova, R. Harrex, J. Røe, K. Rissanen, W. Shi, S. Styring, C. Tommos, K. Warncke and B. R. Wood, Acta Chem. Scand., 1997, 51, 343-344. D. Wang, Z. Yang, C. Wang, A. Zhang and M. Uchiyama, Angew. Chemie Int. Ed., 2018, 57, 3641-3645. D. Y. Wang, X. Wen, C. D. Xiong, J. N. Zhao, C. Y. Ding, Q. Meng, H. Zhou, C. Wang, M. Uchiyama, X. J. Lu and A. Zhang, iscience, 2019, 15, 307-315. T. Uemura, M. Yamaguchi and N. Chatani, Angew. Chemie Int. Ed., 2016, 55, 3162-3165. E. Wenkert, A.-L. Han and C.-J. Jenny, J. Chem. Soc., Chem. Commun., 1988, 975-976. J. T. Reeves, D. R. Fandrick, Z. Tan, J. J. Song, H. Lee, N. K. Yee and C. H. Senanayake, Org. Lett., 2010, 12, 4388-4391. S. B. Blakey and D. W. C. MacMillan, J. Am. Chem. Soc. 2003, 125, 6046-6047. H. Sun and S. G. DiMagno, J. Fluor. Chem., 2007, 128, 806812. N.-U. H. Khan, B. C. Lee, S.-Y. Lee, Y. S. Choe, C.-H. Jun and D. Y. Chi, J. Label. Compd. Radiopharm., 2002, 45, 10451053. R. Richarz, P. Krapf, F. Zarrad, E. A. Urusova, B. Neumaier and B. D. Zlatopolskiy, Org. Biomol. Chem., 2014, 12, 80948099.

X. Huang, R. J. Gillies and H. Tian, J. Label. Compd. Radiopharm., 2015, 58, 156-162. F. Silbestri, M. T. Lockhart and A. B. Chopra, ARKIVOC 2011, 210-220. Y.-T. Wang, B.-B. Gao, F. Wang, S.-Y. Liu, H. Yu, W.-H. Zhang and J.-P. Lang, Dalt. Trans., 2017, 46, 1832-1839. B.-B. Gao, M. Zhang, X.-R. Chen, D.-L. Zhu, H. Yu, W.-H. Zhang and J.-P. Lang, Dalt. Trans., 2018, 47, 5780-5788. J. Martínez, F. de la Cruz-Martínez, M. A. Gaona, E. PinillaPeñalver, J. Fernández-Baeza, A. M. Rodríguez, J. A. CastroOsma, A. Otero and A. Lara-Sánchez, Inorg. Chem., 2019, 58, 3396-3408. S. P. Desai, M. Mondal and J. Choudhury, Organometallics, 2015, 34, 2731-2736. F. Sadegh, O. Bagheri, M. Moghadam, V. Mirkhani, S. Tangestaninejad and I. Mohammadpoor-Baltork, J. Organomet. Chem., 2014, 759, 46-57.
Elliott, M. A. Cooper, J. Perera, S. Swift, I. Blakey, A. K. Whittaker and H. Peng, J. Polym. Sci. Part A Polym. Chem., 2019, 57, 1908-1921.

Y. Ma, L. Zhang, Y. Luo, M. Nishiura and Z. Hou, J. Am. Chem. Soc., 2017, 139, 12434-12437.

Q. Chen, F. Gao, H. Tang, M. Yao, Q. Zhao, Y. Shi, Y. Dang and C. Cao, ACS Catal., 2019, 9, 3730-3736.

L. G. Xie and Z. X. Wang, Angew. Chemie - Int. Ed., 2011, 50, 4901-4904.

Z.-X. Wang and B. Yang, Org. Biomol. Chem., 2020, 18, 1057-1072.

C. Wang, Chem. Pharm. Bull., 2020, 68, 683-693. H. Chen, H. Yang, N. Li, X. Xue, Z. He and Q. Zeng, Org. Process Res. Dev., 2019, 23, 1679-1685.

L. Liu, W.-Q. Yu, T. Huang and T. Chen, Tetrahedron Lett., 2020, 61, 151647.

X.-Q. Zhang and Z.-X. Wang, J. Org. Chem., 2012, 77, 36583663.

J. Hu, H. Sun, W. Cai, X. Pu, Y. Zhang and Z. Shi, J. Org. Chem., 2016, 81, 14-24.

H. Zhang, S. Hagihara and K. Itami, Chem. - A Eur. J., 2015, 21, 16796-16800.

X.-Q. Zhang and Z.-X. Wang, Org. Biomol. Chem., 2014, 12 1448-53.

F. Zhu, J.-L. Tao and Z.-X. Wang, Org. Lett., 2015, 17, 49264929.

R. W. Hoffmann, Angew. Chemie - Int. Ed., 2000, 39, 20542070.

M. Burns, S. Essafi, J. R. Bame, S. P. Bull, M. P. Webster, S. Balieu, J. W. Dale, C. P. Butts, J. N. Harvey and V. K. Aggarwal, Nature, 2014, 513, 183-188.

H. Schönherr and T. Cernak, Angew. Chemie - Int. Ed., 2013, 52, 12256-12267.

E. J. Barreiro, A. E. Kümmerle and C. A. M. Fraga, Chem. Rev., 2011, 111, 5215-5246.

M. Butters, D. Catterick, A. Craig, A. Curzons, D. Dale, A. Gillmore, S. P. Green, I. Marziano, J.-P. P. Sherlock, W. White, K. S. Beistle, D. A. Smith and G. Nagel, Chem. Rev., 2006, 106, 3002-3027.

K. Endo and T. Shibata, Synthesis (Stuttg)., 2012, 44, 14271452.

J. Kim and S. H. Cho, Synlett, 2016, 27, 2525-2529.

R. Giri, S. Thapa and A. Kafle, Adv. Synth. Catal., 2014, 356, 1395-1411.

G. Lamoureux and C. Agüero, Arkivoc, 2009, 2009, 251264.

J. Macgee, J. Anal. Chem., 1970, 42, 421-422.

D. G. Bailey, J. Chromatogr., 1975, 11, 305-311.

L. Hevesi, Tetrahedron Lett., 1976, 17, 1389-1390.

J. L. Day, P. Grenier and L. Harold, J. Chromatogr. A, 1979, 177, 118-121. WO/2018/157233, 2018. US4425350A, 1980. US20050261500A1, 2004.

G. Noonan and A. G. Leach, Org. Biomol. Chem., 2015, 13, 2555-2560.

A. L. Dunn, A. Payne, P. R. Clark, C. McKay, G. D. Williams, 
K. Wheelhouse, K. Arendt, F. Dixon and S. Shilcrat, J. Chem. Educ., 2020, acs.jchemed.0c00115.

53 J. P. Lewicki, D. Todd, P. Redon, J. Liggat and L. Gibson, MRS Proc., 2007, 1047, 1047-Y04-05.

54 P. Storoniak and J. Rak, J. Therm. Anal. Calorim., 2000, 60, 927-941.

55 R. F. Rodewald, K. Mahendran, J. L. Bear and R. Fuchs, J. Am. Chem. Soc., 1968, 90, 6698-6700.

56 W. M. Weaver and J. D. Hutchison, J. Am. Chem. Soc., 1964, 86, 261-265.

57 P. S. Pregosin, Magn. Reson. Chem., 2017, 55, 405-413.

58 E. V. Dalessandro and J. R. Pliego, Theor. Chem. Acc., 2020, 139, 27.

59 E. M. Arnett and D. R. McKelvey, J. Am. Chem. Soc., 1966, 88, 2598-2599.

60 E. Avella-Moreno, N. Nuñez-Dallos, L. Garzón-Tovar and A. Duarte-Ruiz, J. Sulfur Chem., 2015, 36, 535-543.

61 E. E. Kwan, Y. Zeng, H. A. Besser and E. N. Jacobsen, Nat. Chem., 2018, 10, 917-923.

62 C. Gardner Swain and E. C. Lupton, J. Am. Chem. Soc., 1968, 90, 4328-4337.

63 J. T. Burns and K. T. Leffek, Can. J. Chem., 1969, 47, 37253728. S. D. Roughley and A. M. Jordan, J. Med. Chem., 2011, 54, 3451-3479.

65 D. G. Brown and J. Boström, J. Med. Chem., 2016, 59, 4443-4458.

66 M. J. Frisch, G. W. Trucks, H. B. Schlegel, G. E. Scuseria, M. A. Robb, J. R. Cheeseman, G. Scalmani, V. Barone, B. Mennucci, G. A. Petersson, H. Nakatsuji, M. Caricato, X. Li, H. P. Hratchian, A. F. Izmaylov, J. Bloino, G. Zheng, J. L. Sonnenberg, M. Hada, M. Ehara, K. Toyota, R. Fukuda, J. Hasegawa, M. Ishida, T. Nakajima, Y. Honda, O. Kitao, H. Nakai, T. Vreven, J. J. A. Montgomery, J. E. Peralta, F.

Ogliaro, M. Bearpark, J. J. Heyd, E. Brothers, K. N. Kudin, V. N. Staroverov, R. Kobayashi, J. Normand, K. Raghavachari, A. Rendell, J. C. Burant, S. S. Iyengar, J. Tomasi, M. Cossi, N. Rega, J. M. Millam, M. Klene, J. E. Knox, J. B. Cross, V. Bakken, C. Adamo, J. Jaramillo, R. Gomperts, R. E. Stratmann, O. Yazyev, A. J. Austin, R. Cammi, C. Pomelli, J. W. Ochterski, R. L. Martin, K. Morokuma, V. G. Zakrzewski, G. A. Voth, P. Salvador, J. J. Dannenberg, S. Dapprich, A. D. Daniels, O. Farkas, J. B. Foresman, J. V. Ortiz, J. Cioslowski and D. J. Fox, 2009. G. Luchini, J. V. Alegre-Requena, I. Funes-Ardoiz and R. S. Paton, F1000Research, 2020, 9, 291. 